DESY $14-071$

ISSN $0418-9833$

May 2014

\title{
Spin Transport and Polarimetry in the Beam Delivery System of the International Linear Collider
}

\author{
M. Beckmann ${ }^{1,2}$, J. List ${ }^{1}$, A. Vauth ${ }^{1,2}$, and B. Vormwald ${ }^{1,2}$. \\ 1- Deutsches Elektronen-Synchrotron DESY \\ Notkestr. 85 \\ 22607 Hamburg, Germany \\ 2- University of Hamburg \\ Institute for Experimental Physics \\ Luruper Chaussee 149 \\ 22761 Hamburg, Germany
}

\begin{abstract}
Polarised electron and positron beams are key ingredients to the physics programme of future linear colliders. Due to the chiral nature of weak interactions in the Standard Model - and possibly beyond - the knowledge of the luminosity-weighted average beam polarisation at the $e^{+} e^{-}$interaction point is of similar importance as the knowledge of the luminosity and has to be controlled to permille-level precision in order to fully exploit the physics potential. The current concept to reach this challenging goal combines measurements from Laser-Compton polarimeters before and after the interaction point with measurements at the interaction point. A key element for this enterprise is the understanding of spin-transport effects between the polarimeters and the interaction point as well as collision effects. We show that without collisions, the polarimeters can be cross-calibrated to $0.1 \%$, and we discuss in detail the impact of collision effects and beam parameters on the polarisation value relevant for the interpretation of the $e^{+} e^{-}$collision data.
\end{abstract}




\section{Introduction}

Beam polarisation is a key ingredient to the physics programme of future linear colliders. In particular the International Linear Collider [1] foresees in its baseline configuration longitudinal polarisation of the electron and positron beams of $\mathcal{P}_{z}= \pm 80 \%$ and $\pm 30 \%$, respectively. Upgrade options comprise a higher degree of positron polarisation of up to $\pm 60 \%$.

Due to the chiral nature of weak interactions in the Standard Model, most crosssections depend linearly on the longitudinal beam polarisations, rendering the knowledge of the luminosity-weighted average polarisations at the $e^{+} e^{-}$interaction point as important as the knowledge of the luminosity itself. This does primarily apply to electroweak precision measurements and indirect searches for new physics, e.g. via anomalous couplings of the top quark 2, 3]. Moreover, it also concerns direct searches for small signals above large irreducible backgrounds, e.g. WIMP Dark Matter searches in the mono-photon signature [4]. While the luminosity is expected to be measured to a few permille based on small-angle Bhabha scattering [5], permille-level precision is challenging for polarimetry. The design goal is thus to reach $\delta \mathcal{P}_{z} / \mathcal{P}_{z}=0.25 \%$, while physics would profit further if $0.1 \%$ could be reached.

The overall concept to determine the beam polarisation is based on the interplay of several complementary approaches: Laser-Compton polarimeters will monitor the instantaneous polarisation in some distance from the $e^{+} e^{-}$interaction point (IP). Their fast measurements give important feed-back to the accelerator operators, but also track time variations on longer time scales as well as possible patterns within a bunch train. On each beam, there will be one polarimeter $\sim 1650 \mathrm{~m}$ upstream, and a second one $\sim 150 \mathrm{~m}$ downstream of the IP. While the upstream polarimeter measures the initial polarisation under very clean conditions, the downstream polarimeter serves a double purpose: In absence of collisions, the polarimeters can be cross-calibrated, if the spin transport between both locations can be predicted with sufficient precision, i.e. to $0.1 \%$ or better. In collision mode, the depolarising effects of the beam-beam interaction and the luminosity-weighted average polarisation at the IP could be monitored. This enterprise, however, depends crucially on a sufficiently precise understanding of all effects of the beam-beam interaction and the spin transport, which is the main objective of this publication.

The luminosity-weighted average polarisation at the IP can be directly accessed from $e^{+} e^{-}$collision data themselves. Several approaches have been studied for this in the past [6 8], comprising schemes operating on the measurements of total cross-sections for various polarisation configurations, as well as on single- and double-differential distributions of $W^{+} W^{-}$production. All these approaches will finally yield a very important long-term scale calibration of the luminosity-weighted average polarisation when appropriately compared with the polarimeter measurements. However, they all will take years of data taking before reaching permille-level precision. Furthermore, they assume that the helicity reversal is exact, i.e. that the absolute value of the polarisation is the same for all helicity configurations. Any deviation from this assumption can only be corrected for based on the polarimeter measurements and their propagation to the IP. It has been shown that the need for such a correction could limit the precision of the collision data 
methods at the precision of the polarimeters [7]. The difference in absolute polarisation values between data sets, however, can be minimized if the helicity of both beams can be reversed quickly and independently, e.g. on a pulse-by-pulse basis [9]. For the electron beam, this is readily provided by switching the helicity of the source laser. For the positron beam, currently a scheme based on switching between two spin rotator beam lines as proposed in [10] is foreseen [1].

This paper is organised as follows: Sections 2 and 3 introduce the beam delivery system of the ILC and the spin-tracking formalism, respectively. The design and the capabilities of the Laser-Compton polarimeters will be summarised in section 4. In section 5 we will study the achievable precision for cross-calibration of the polarimeters in the absence of collisions, while section 6 discusses the extraction of the luminosity-weighted average polarisation in the presence of collisions. We conclude in section 7 with the prospects for reaching the goal of a few permille precision and give an outlook on studies required in the future.

\section{Accelerator Environment}

In this section the parts of the ILC most relevant for the determination of the luminosityweighted average polarisation at the $e^{+} e^{-}$interaction point will be introduced. This includes the beam delivery systems and the extraction lines as well as the different beam parameter sets used in the simulation study. Since most of the aspects studied in this paper are identical between electrons and positrons, we will use the term "electron" for both beams, unless specific differences need to be pointed out.

\subsection{The ILC Beam Delivery System and Extraction Line}

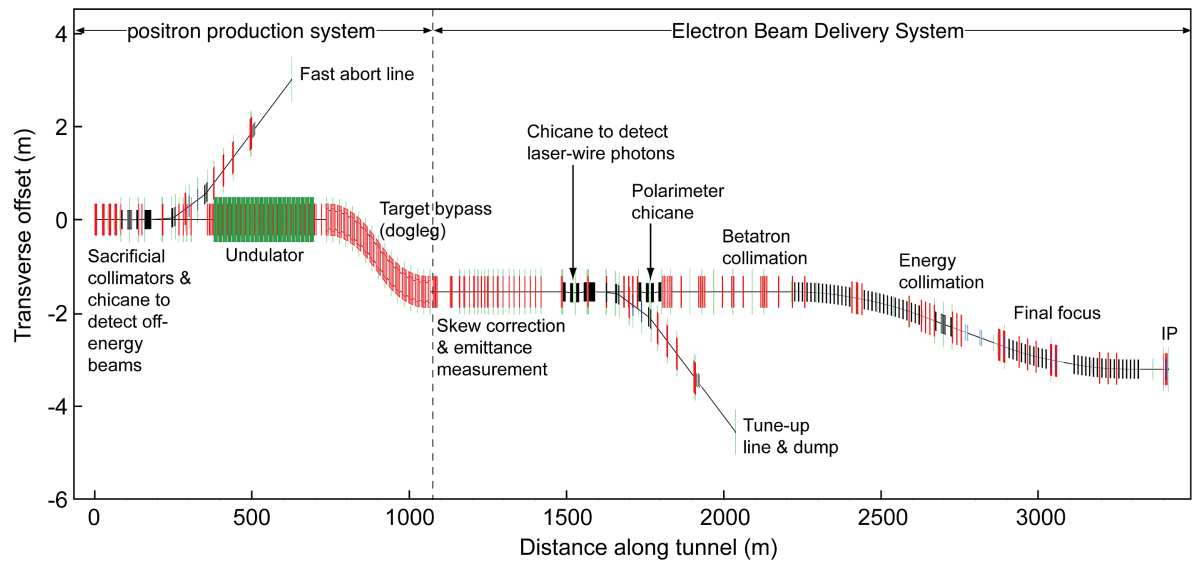

Figure 1: Layout of the electron beam delivery system (BDS). The positron BDS is a mirror image. From figure 2.12 in $[11$.

The beam delivery system (BDS) is an about $2 \mathrm{~km}$ long set of beamlines which serves the final preparation of the fully accelerated beams for collisions. It hosts beam diagnostics, skew correction, betatron and energy collimation as well as the final focus system, 
and has been carefully designed to minimise the emittance growth. This applies in particular to the vertical direction, since flat beams are essential to reconcile high luminosity with minimal beamstrahlung [12]. Therefore, all bends in the BDS are in the horizontal plane. Figure 1 shows a sketch of the BDS for the electron beam up to the $e^{+} e^{-}$IP. The upstream polarimeter is located directly behind the branch-off to the tune-up dump. The positron BDS is a mirror image, apart from the positron production system (left part of figure 1).

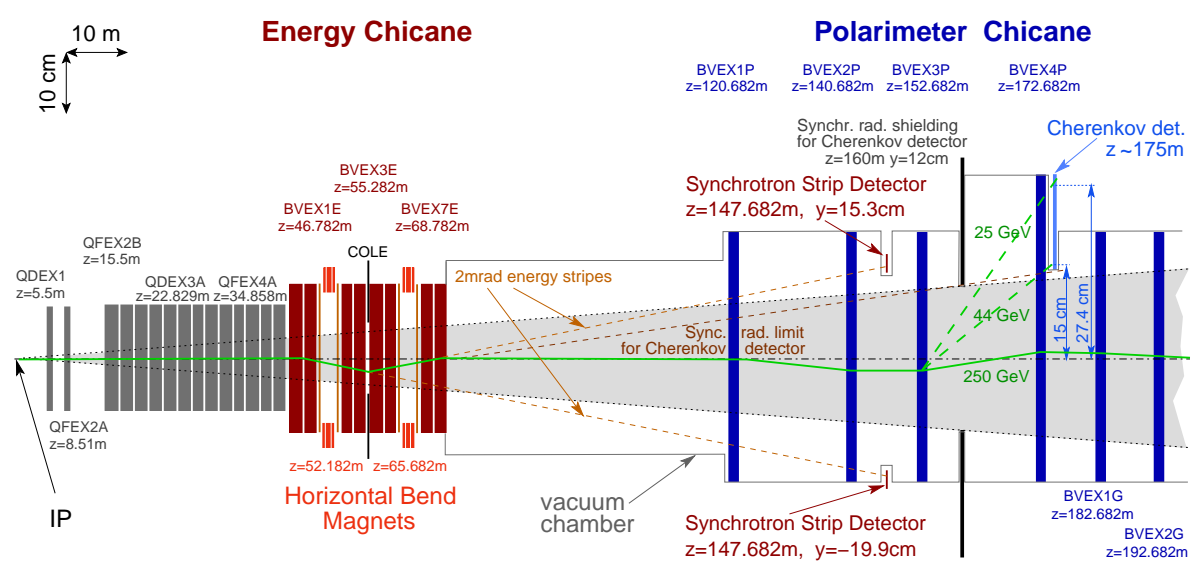

Figure 2: Schematic view of the extraction line up to the downstream polarimeter. From $[13$.

Figure 2 shows the extraction line, which guides the spent beams to the dumps and provides post-collision diagnostics. In particular, the downstream polarimeter is located at a secondary focus point of the optics, in order to provide the best possible beam conditions at the Compton IP of the downstream polarimeter.

\subsection{Beam Parameters and Interaction Region}

Table 1 lists the design beam parameters at the IP according to the Reference Design Report (RDR) [14] and the more recent Technical Design Report (TDR) [1. Most of the studies presented in this paper have been performed with the RDR parameters, since they match the available lattic $\mathrm{e}^{1}$. For the current TDR parameter set, the number of bunches per train is reduced with respect to the RDR. In order to restore the luminosity, the beams are focussed more strongly at the IP. This results in more intense collisions, which might also affect the polarsation. Therefore, the collision effects and the spin transport to the downstream polarimeter have been studied for both parameter sets. The electron and positron beam parameters are identical apart from the beam energy spread $\sigma_{E} / E$, which is slightly increased for the electrons during their passage through the undulator of the positron source.

The ILC design foresees a horizontal crossing angle of $14 \mathrm{mrad}$ between the $e^{-}$and the $e^{+}$beamlines at the IP. In order to maximise the luminosity, the bunches are correspondingly rotated by $7 \mathrm{mrad}$ using crab cavities [15].

\footnotetext{
${ }^{1}$ The lattice describes the layout of a beamline, e.g. positions and strengths of the magnets.
} 


\begin{tabular}{|c|c|c|c|c|}
\hline Parameter & Symbol & & RDR & TDR \\
\hline Bunches per train & & & 2625 & 1312 \\
\hline Train frequency & & {$[\mathrm{Hz}]$} & 5 & 5 \\
\hline Horizontal bunch size & $\sigma_{x e}$ & [nm] & 639 & 474 \\
\hline Vertical bunch size & $\sigma_{y e}$ & {$[\mathrm{~nm}]$} & 5.7 & 5.9 \\
\hline Horizontal angular spread & $\theta_{x}$ & {$[\mu \mathrm{rad}]$} & 32 & 43 \\
\hline Vertical angular spread & $\theta_{y}$ & {$[\mu \mathrm{rad}]$} & 14 & 12 \\
\hline Horizontal norm. emittance & $\gamma \varepsilon_{x}$ & {$[\mu \mathrm{m}]$} & 10 & 10 \\
\hline Vertical norm. emittance & $\gamma \varepsilon_{y}$ & {$[\mathrm{~nm}]$} & 40 & 35 \\
\hline Horizontal disruption parameter & $D_{x}$ & & 0.17 & 0.3 \\
\hline Vertical disruption parameter & $D_{y}$ & & 19.4 & 24.6 \\
\hline Beam energy spread $\left(e^{-}, e^{+}\right)$ & $\sigma_{E} / E$ & {$\left[10^{-3}\right]$} & $1.4,1.0$ & $1.24,0.7$ \\
\hline$e^{+} e^{-}$luminosity & $\mathcal{L}$ & {$\left[10^{34} \mathrm{~cm}^{-2} \mathrm{~s}^{-1}\right]$} & 2 & 1.8 \\
\hline
\end{tabular}

Table 1: Selected beam parameters at the IP for $E_{c m}=500 \mathrm{GeV}$ according to RDR [16 (nominal parameter set) and TDR [11] (baseline parameters).

The collider experiments at the IP contain two types of magnets which also influence the beams. These are the main solenoid for the tracking system and the anti-DID (detector-integrated dipole) to guide electron-positron pairs produced by beamstrahlung photons into the outgoing beam pipe [17]. Due to the crossing angle, the detector magnets at the IP are rotated by $7 \mathrm{mrad}$ with respect to each of the beamlines. These magnets are not yet present in the official lattice files, but have been included in our simulations by hand.

\subsection{Magnet Misalignments and Orbit Correction}

Misalignments of the magnets in an accelerator have various effects, among others beam jitter (deviations of the beam orbit from the design orbit) or a detuned focussing. There are static misalignments due to the limited precision to which the beamline elements can be adjusted at their designated positions, and time-dependent misalignments from various sources, e.g. from seismic noise, traffic, or cooling water pumps.

To minimise the beam jitter and prevent a beam loss in the worst case, the ILC will be equipped with several feedback orbit correction systems. These systems consist of dipole magnets to perform the orbit correction and of beam position monitors, which measure the current beam position from which the required field strengths for the correction magnets are recalculated. They operate on a timescale of a tenth of a second, i.e. the measurements from one bunch train are used to correct the orbit for the next bunch train. At the IP, a higher precision is required to bring the beams with vertical sizes of few nanometers to collision with the envisaged luminosity. Therefore, an additional fast-feedback system is foreseen at the IP, which operates on a timescale of nanoseconds, i.e. bunch-to-bunch. The interplay of ground motion and the fast-feedback system and their effects on luminosity and polarisation have been studied previously [18, 19]. It has been shown in particular that without correction, ground-motion-induced misalignments 
and the resulting depolarisation become significant only at time scales of a day. Thus, the feedback required for maintaining luminosity is considered sufficient to also maintain polarisation, and we will not study time-dependent misalignments in this publication.

\section{Spin Transport and Collision Effects}

In this section, the basic effects on the polarisation which occur in the beam delivery system, the interaction region, and the extraction line as well as their implementation in the simulation are introduced.

\subsection{Spin Transport}

The spins of particles in an accelerator are subject to spin precession in electromagnetic fields and to spin-flips from the emission of photons. For the bunch propagation through the ILC beam delivery system, it turns out that the effects of spin-flips can be neglected (cf. section 5). The energy loss due to synchrotron radiation, however, affects the particle trajectories and the spin precession downstream.

The change of a particle spin vector $\vec{S}$ with time under the influence of electromagnetic fields is described by the Thomas-Bargmann-Michel-Telegdi (T-BMT) equation [20, 21]. Since neither the beam delivery system nor the extraction line contains components with sizable electric fields, the full T-BMT equation simplifies to

$$
\frac{d}{d t} \vec{S}=\vec{\Omega}_{B}(\vec{B}, \vec{r}, \vec{p}, t) \times \vec{S}=-\frac{q}{m \gamma}\left((1+a \gamma) \vec{B}-\frac{a \vec{p} \cdot \vec{B}}{(\gamma+1) m^{2} c^{2}} \vec{p}\right) \times \vec{S}
$$

Here, $\vec{B}(\vec{r}, t)$ denotes the magnetic field, $\gamma$ the relativistic Lorentz factor, $c$ the vacuum speed of light and $a \equiv(g-2) / 2$ the anomaly of the gyro-magnetic moment, with $a \approx$ 0.001159652 for electrons.

The expression for $\vec{\Omega}_{B}$ in equation 1 can be decomposed in two parts for the field components, $\vec{B}_{\|}$parallel to $\vec{p}$ and $\vec{B}_{\perp}$ perpendicular to it:

$$
\vec{\Omega}_{B}(\vec{B}, \vec{r}, \vec{p}, t)=-\frac{q}{m \gamma}\left((1+a \gamma) \vec{B}_{\perp}+(1+a) \vec{B}_{\|}\right)
$$

In presence of only perpendicular magnetic fields, the momentum $\vec{p}$ and the spin $\vec{S}$ behave very similarly:

$$
\begin{aligned}
\frac{d}{d t} \vec{p} & =-\frac{q}{m \gamma}\left(\begin{array}{r}
\left.\vec{B}_{\perp}\right) \times \vec{p} \\
\frac{d}{d t} \vec{S}
\end{array}=-\frac{q}{m \gamma}\left((1+a \gamma) \vec{B}_{\perp}\right) \times \vec{S}\right.
\end{aligned}
$$


Thus, the spin vector precesses in a perpendicular magnetic field about $\vec{B}$ by the angle

$$
\xi_{\text {spin }}=(1+a \gamma) \xi_{\text {orbit }}
$$

where $\xi_{\text {orbit }}$ is the deflection angle of the particle [22,23]. For an electron beam with an energy of $250 \mathrm{GeV}$, the amplification factor is $(1+a \gamma) \approx 568$; for an energy of $500 \mathrm{GeV}$, it rises to $\approx 1136$.

For a particle beam with a spatial extension, an angular divergence and an energy spread in an inhomogeneous magnetic field, the spin precessions for different particles can vary significantly. One possible pattern emerging from non-uniform spin precession is referred to as "spin fan-out" in the following and occurs in inhomogeneous magnetic fields or in the presence of a beam energy spread. Figure 3 illustrates spin fan-out and its reversability using the example of a hypothetical two-particle bunch traversing quadrupole magnets: Both particles are deflected into different directions in the first quadrupole and the spin vectors precess correspondingly according to equation 5. Thus, the longitudinal polarisation decreases, but the transverse polarisation remains zero since the transverse components of the two spin vectors cancel each other. Consequently, the magnitude of the polarisation decreases: $\left|\overrightarrow{\mathcal{P}}^{\prime}\right|<|\overrightarrow{\mathcal{P}}|$. This can in principle be reversed by a second quadrupole which rotates the spin vectors back to the original orientation.

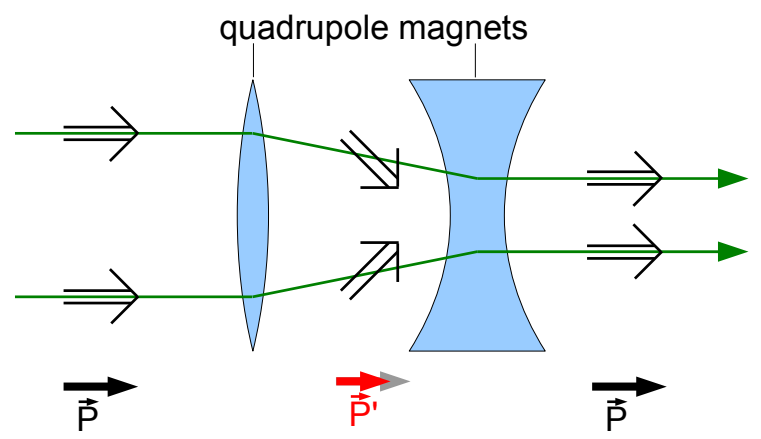

Figure 3: Spin fan-out at the example of two hypothetical particles. This sketch only illustrates the possible effects of quadrupole magnets on the beam polarisation. It does not describe realistic focussing, the two shown quadrupole magnets do not have the same strength, and betatron oscillations in two dimensions are not indicated. 24]

In a beamline consisting of F0D0 cell: $2^{2}$, however, these spin precessions occur alternatingly in the horizontal and the vertical plane. Rotations in two dimensions do generally not commute, but this fact can be neglected if the rotations are sufficiently small. In that case, the spin fan-out can be set into relation to the angular divergence $\theta_{r}$ (i.e. to the fan-out of the momentum vectors) of the bunch by the following function, assuming a longitudinally polarised beam, for which the maximum polarisation $|\overrightarrow{\mathcal{P}}|_{\max }$ is obtained for $\theta_{r}=0$ (as in figure 3):

$$
f\left(\theta_{r}\right)=|\overrightarrow{\mathcal{P}}|_{\max } \cdot \cos \left((1+a\langle\gamma\rangle) \cdot \theta_{r}\right),
$$

\footnotetext{
${ }^{2}$ Standard set of focussing (F) and defocussing (D) quadrupoles interleaved with drift spaces (0).
} 
where $\langle\gamma\rangle$ denotes the average relativistic Lorentz factor.

In presence of a beam energy spread, a similar behavior occurs (also in homogeneous magnetic fields), since the "amplification factor" $(1+a \gamma)$ is energy-dependent. This fanout is in principle reversible as well.

So it might seem that polarisation can be generated by spin fan-out, but that is actually not the case. Spin fan-out allows only to restore an existing ordering in the spin orientation which is preserved in a correlation between the spin orientation and the particle energy or a particle coordinate. It is not possible to restore polarisation which got lost in stochastic processes like radiative depolarisation, which will be introduced in the following section.

The description of the spin fan-out by equation 6, however, relies on sufficiently small rotations. Verifying the validity of this assumption for the ILC BDS is one of the objectives of a full spin tracking study.

\subsection{Beam-Beam Collisions and Luminosity-Weighted Average Polarisation}

In the beam-beam collisions at the IP, the colliding bunches distort each other by their electromagnetic fields, while only a few particles undergo hard interactions. The polarisation of these particles is the luminosity-weighted polarisation for a single collision of an electron bunch with a positron bunch, which will be denoted here by the symbol $\overrightarrow{\mathcal{P}}^{\text {lumi, } 1}$. It is luminosity-weighted with respect to the local distribution of the luminosity during a collision.

The decisive quantity for interpreting collision data is the luminosity-weighted polarsation averaged over time $\overrightarrow{\mathcal{P}}^{\text {lumi }}$ for each of the beams, defined as

$$
\overrightarrow{\mathcal{P}}^{\text {lumi }}=\frac{\int \mathcal{L}(t) \overrightarrow{\mathcal{P}}^{\text {lumi }, 1}(t) d t}{\int \mathcal{L}(t) d t},
$$

and in particular its longitudinal component $\mathcal{P}_{z}^{\text {lumi }}$. Depending on the chiral structure of the studied observable, it might also be advantageous to consider the time average of one of the effective polarisations [6] listed in table 2 instead, which can all be defined in analogy to equation 7. The gain in error reduction when using the appropriate effective

\begin{tabular}{c|l|l} 
effective polarisation & observable & type of interaction \\
\hline $\mathcal{P}_{e^{-+}} \mathcal{P}_{e^{+}}$ & $A_{L R}$ & $s$-channel vector exchange \\
$1+\mathcal{P}_{e^{-}} \mathcal{P}_{e^{+}}$ & cross-section & $s$-channel vector exchange \\
$\mathcal{P}_{e^{-}} \mathcal{P}_{e^{+}}$ & cross-section & $t$-channel $W$ or $\nu_{e}$ production \\
$\mathcal{P}_{e^{-}}+\mathcal{P}_{e^{+}}-\mathcal{P}_{e^{-}} \mathcal{P}_{e^{+}}$ & croduction
\end{tabular}

Table 2: Effective polarisations minimising the impact of polarisation uncertainties on various observables.

polarisations depends on the degree of correlation between the measurements of electron 
and positron polarisation. In this respect, it is reasonable to assume that the instrumental uncertainties of the polarimeters as well as the influence of misalignments in the BDS are uncorrelated. Collision effects on the other hand have to be assumed to be highly correlated, since the intensity of the collision is reciprocal.

In head-on collisions, the already focussed bunches attract each other even further due to their mutual electrical fields (pinch effect [12]). The T-BMT precession due to this mutual focussing of the bunches leads to a spin fan-out like in a quadrupole magnet. For flat, longitudinally polarised beams $\left(\sigma_{x e} \gg \sigma_{y e}, \mathcal{P}_{z}=|\overrightarrow{\mathcal{P}}|\right)$ and a small horizontal disruption parameter $\left(D_{x} \ll 1\right)$, the polarisations before $\left(|\overrightarrow{\mathcal{P}}|^{\text {bef }}\right)$ and after $\left(|\overrightarrow{\mathcal{P}}|^{\text {aft }}\right)$ the collision as well as the luminosity-weighted polarisation $|\overrightarrow{\mathcal{P}}|^{\text {lumi,1 }}$ are related as follows (equation 16 in [25]):

$$
|\overrightarrow{\mathcal{P}}|^{\text {bef }}-|\overrightarrow{\mathcal{P}}|^{\text {lumi,1 }}=0.273\left(|\overrightarrow{\mathcal{P}}|^{\text {bef }}-|\overrightarrow{\mathcal{P}}|^{\text {aft }}\right)
$$

If the angular divergence of the bunches before the collision is negligible in terms of spin fan-out, the spin fan-out during the collision can be related to the angular divergence $\theta_{r}^{\text {aft }}$ after the collision (equation 31 in [25], see also equation 6]:

$$
|\overrightarrow{\mathcal{P}}|^{\text {bef }}-|\overrightarrow{\mathcal{P}}|^{\text {aft }} \approx \frac{1}{2}|\overrightarrow{\mathcal{P}}|^{\text {bef }} \cdot(1+a \gamma)^{2} \cdot\left(\theta_{r}^{\text {aft }}\right)^{2}
$$

Merging these two equations, one obtains:

$$
|\overrightarrow{\mathcal{P}}|^{\text {bef }}-|\overrightarrow{\mathcal{P}}|^{\text {lumi, }, 1} \approx \frac{1}{2}|\overrightarrow{\mathcal{P}}|^{\text {bef }} \cdot(1+a \gamma)^{2} \cdot\left(\frac{\theta_{r}^{\text {aft }}}{2}\right)^{2}
$$

As explained in [26] and [27], one can interpret this as about half of the T-BMT precession occurring before the hard interaction. A comparison to equation 9 implies that one can reproduce the luminosity-weighted polarisation at a point behind the IP where the angular divergence has to be reduced by a factor $1 / 2$ with respect to the divergence at the IP after the collision.

During the mutual distortion of the bunches, the deflected particles also emit beamstrahlung photons, which can cause a flip of the electron spin by the Sokolov-Ternov effect [28. Unlike for the synchrotron radiation in the BDS, the radiative depolarisation in the collision could reach a non-negligible level. Therefore, dedicated simulations of the bunch-bunch interaction are needed in order to verify whether equation 10 holds for realistic ILC beam parameters. A detailed description of the beamstrahlung-related effects on the polarisation can be found in section 5.9 of [29].

\subsection{Simulation Framework STALC}

In order to study the spin transport in the beam delivery system of a linear collider, the simulation framework STALC (Spin Transport at Linear Colliders) has been developed [24]. As sketched in figure 4, it interfaces dedicated programs to simulate the beam transport 


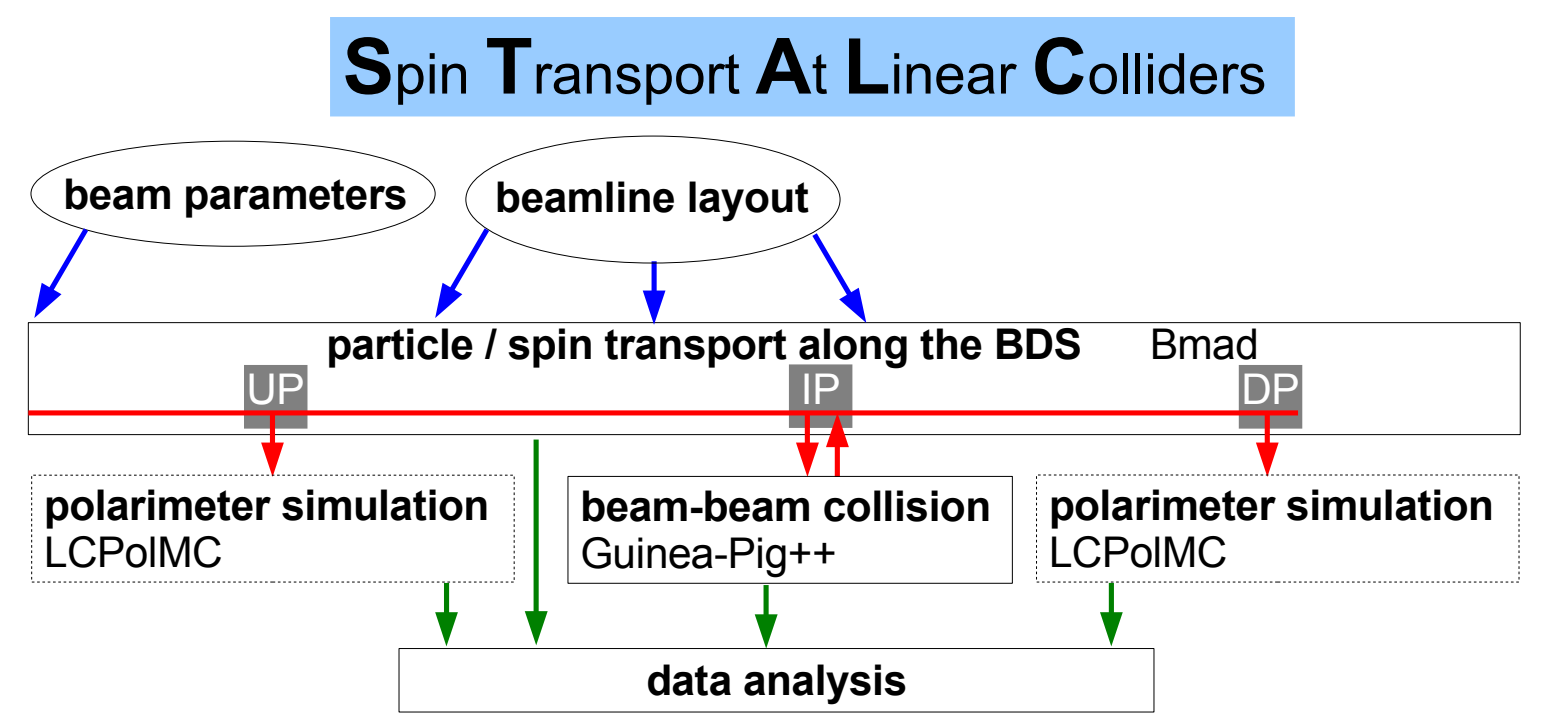

Figure 4: Program flow in STALC. UP/DP denotes the up-/downstream polarimeter.

including the polarisation through the BDS, the collisions at the IP and the measurements of the polarimeters, respectively:

STALC generates electron/positron bunches and tracks them through a given lattice via Bmad [30, 31], a subroutine library for particle simulations in high-energy accelerators, which can take also the polarisation into account. At the polarimeters, the particle information can be passed to LCPolMC, which simulates the polarisation measurement in a Compton polarimeter from the Compton scattering process to the detector response. At the IP, the particle information from one electron bunch and one positron bunch is passed to Guinea-Pig++ [32] to simulate the beam-beam collisions at the IP. Guinea-Pig++ is an extension of Guinea-Pig [33], which takes into account the polarisation and simulates T-BMT precession and Sokolov-Ternov effects. The spent bunches after the collision are passed back to the accelerator simulation and tracked to the downstream polarimeter. The final analysis is based on ROOT 34.

STALC is not inherently limited to the ILC, but can be run on any lattice and beam parameter set.

\section{Compton Polarimetry at the ILC}

In this section, we will summarise the working principle and the capabilities of the LaserCompton polarimeters at the ILC. A more detailed description of both polarimeters can be found in $[13]$.

The single differential cross section $d \sigma / d E$ for Compton scattering 35] contains a term proportional to the product of the longitudinal beam polarisation and the circular laser 
polarisation, which can be isolated by measuring the count-rate asymmetry with respect to the laser helicity.

In order to quickly gather statistical precision, both polarimeters operate in a multievent mode with $\mathcal{O}\left(10^{3}\right)$ Compton interactions per bunch.

\begin{tabular}{lccc} 
& $e^{+} / e^{-}$beam & \multicolumn{2}{c}{ Laser beam } \\
& & Upstream & Downstream \\
\hline Energy & $45.6-500 \mathrm{GeV}$ & $2.33 \mathrm{eV}$ & $2.33 \mathrm{eV}$ \\
Bunch charge/energy & $2 \cdot 10^{10} e$ & $35 \mu \mathrm{J}$ & $100 \mathrm{~mJ}$ \\
Bunches per train & $1312-2625$ & $1312-2625$ & 1 \\
Bunch length $\sigma_{t}$ & $1.3 \mathrm{ps}$ & $10 \mathrm{ps}$ & $2 \mathrm{~ns}$ \\
Average power & & $0.2-0.5 \mathrm{~W}$ & $0.5 \mathrm{~W}$
\end{tabular}

Table 3: Selected parameters of the electron and laser beams at the up- and downstream polarimeter.

Table 3 summarises specifications of the electron and laser beams relevant for the luminosity calculation. A striking difference between the up- and downstream polarimeter lasers is the energy per bunch, which differs by more than three orders of magnitude. At the location of the downstream polarimeter, significant amounts of background are expected, at the level of $\mathcal{O}\left(10^{3}\right)$ photons and $\mathcal{O}\left(10^{2}\right)$ charged particles per bunch crossing [36]. In order to maintain a suitable signal-to-background ratio in presence of this background, a significantly higher laser power per shot is needed, at the price of a much lower repetition rate and longer pulse durations. This allows to shoot at one electron bunch per train, or a few if several laser are employed. In the nearly background-free conditions of the upstream polarimeter, it is possible to cover every bunch in a train, e.g. by employing similar lasers as for the electron source [37].

\begin{tabular}{lccccccc} 
& & \multicolumn{2}{c}{ UP } & \multicolumn{2}{c}{ DP } & DP with collisions \\
& & $e^{+}$ & $e^{-}$ & $e^{+}$ & $e^{-}$ & $e^{+} / e^{-}$ \\
\hline Horizontal bunch size $\sigma_{x e}$ & {$[\mu \mathrm{m}]$} & 24 & 32 & 7 & 15 & $\sim 3000$ \\
Vertical bunch size $\sigma_{y e}$ & {$[\mu \mathrm{m}]$} & 3 & 3 & 33 & 39 & $\sim 1200$ \\
Beam energy spread $\sigma_{E} / E$ & {$\left[10^{-3}\right]$} & 0.7 & 1.2 & 0.7 & 1.3 & $\sim 44$
\end{tabular}

Table 4: Size and energy spread of electron and positron beams at the up- and downstream polarimeter locations obtained from STALC.

The relevant electron beam sizes at the polarimeter location as obtained from STALC are shown in table 4. It should be noted that after collisions, the beams are highly disrupted and have large non-Gaussian tails, as will be discussed in more detail in section 6.1. Thus, the Gaussian beam sizes listed here should be considered as indicative only.

Table 5 finally shows the actual luminosities per bunch and per second, calculated for laser crossing under a small angle $\alpha$ in the vertical plane according to

$$
\mathcal{L}=\frac{N_{e} N_{\gamma}}{2 \pi \sqrt{\left(\sigma_{x e}^{2}+\sigma_{x \gamma}^{2}\right)} \sqrt{\left(\sigma_{y e}^{2}+\sigma_{y \gamma}^{2}\right)+\left(\sigma_{z e}^{2}+\sigma_{z \gamma}^{2}\right)(\alpha / 2)^{2}}} .
$$




\begin{tabular}{lccc} 
& & Upstream & Downstream \\
\hline Beam crossing angle $\alpha$ & {$[\mathrm{mrad}]$} & 10 & 15.5 \\
Crossing plane & & horizontal & vertical \\
Laser beam size $\sigma_{x \gamma}=\sigma_{y \gamma}$ & {$[\mu \mathrm{m}]$} & 50 & 100 \\
Luminosity / bunch & {$\left[10^{4} \mathrm{~b}^{-1}\right]$} & 1.0 & 18 \\
Luminosity & {$\left[10^{29} \mathrm{~cm}^{-2} \mathrm{~s}^{-1}\right]$} & 630 & 9.1 \\
$\delta \mathcal{P}_{z} / \mathcal{P}_{z}$ (stat) & & $<1 \% / \mathrm{s}$ & $<1 \% / \mathrm{min}$ \\
$\delta \mathcal{P}_{z} / \mathcal{P}_{z}$ (sys) & & $0.25 \%$ & $0.25 \%$
\end{tabular}

Table 5: Selected parameters of the up- and downstream polarimeters. For the downstream polarimeter, the luminosities are based on the electron beam parameters in absence of collisions at the $e^{+} e^{-}$IP.

As desired, the luminosity per bunch turns out to be a factor 20 larger at the downstream polarimeter than at the upstream polarimeter, at the price of a much lower instantaneous luminosity. This in turn leads to a longer time required to reach the same statistical precision. Nevertheless, both measurements are expected to be systematically limited after a very short time.

Since the electron beam is much more energetic than the (optical) laser photons, all scattered particles are forward directed within a narrow cone of $10-20 \mu \mathrm{rad}$. For the relevant photon and electron energies, the total Compton cross section amounts to 154 (118) mb for anti-parallel (parallel) laser and electron polarisation. For the luminositiy of the upstream (downstream) polarimeter, this results in $\mathcal{O}\left(10^{3}\right)\left(\mathcal{O}\left(10^{4}\right)\right)$ Compton scatterings per bunch. The Compton scattered electrons are momentum-analysed by a set of dipole magnets, and their flux is measured as a function of position. The count-rate asymmetry expected for a fully polarised beam is called analysing power. The magnets are arranged as part of a chicane such that the undisturbed beam resumes its original trajectory after passing the whole polarimeter section, while the Compton-scattered electrons should be kicked out sufficiently far from the main beam axis to allow detection.

In case of the upstream polarimeter, the chicane consists of four symmetric sets of dipole magnets, with the Compton interaction point in the middle and the detector behind the forth set of dipoles. Such a configuration offers the additional advantage of decoupling the detector acceptance from the initial beam energy, since the Compton spectrum is projected onto the same area in the detector plane for all beam energies. Instead the dispersion in the middle of the chicane and thus the position of the Compton IP changes according to beam energy.

In case of the downstream polarimeter, the chicane is based on six dipoles, again with the detector behind the forth dipole. In this case, the third and forth dipole are operated at a larger magnetic field in order to kick the Compton signal sufficiently far out of the synchrotron radiation fan from upstream magnets. The fifth and sixth dipole then bring the main beam back to its original trajectory. Detailed specifications for both chicanes can be found in 13$]$.

The lattice between the IP and the downstream polarimeter has been designed such that the angular divergence of the beam at the downstream polarimeter is by a factor 
two smaller than at the end of the collision ${ }^{3}$. Thus, the downstream polarimeter measurement is expected to relate directly to the luminosity-weighted average polarisation during collisions as explained in section 3.2. Whether this picture holds e.g. in presence of beamstrahlung will be investigated in section 6 .

Building on the successful experience at SLD [38], the baseline foresees an array of 20 gas-Cherenkov detectors read-out by photomultiplier tubes [39]. The advantages of this technology comprise radiation hardness, robustness against low-energetic backgrounds due to a Cherenkov threshold in the MeV-regime and a low number of channels to be read-out.

The limiting systematic uncertainties of the SLD polarimeter were the knowledge of the analysing power and the photo detector non-linearities. Both aspects have been studied for the ILC polarimeters in a recent R\&D effort: The dominating contribution to the analysing power, i.e. the alignment of the detector with respect to the beam, has been studied in a testbeam campaign with a prototype [39], and a calibration system for monitoring and correcting non-linearities to a sufficient level has been developed [40]. Based on this experience, the uncertainty budget listed in table 6 seems achievable. Details on the online monitoring of the laser polarisation can be found in [36]. The total systematic uncertainty on the polarimeter measurements amounts to $\delta \mathcal{P}_{z} / \overline{\mathcal{P}}_{z}=0.25 \%$.

\begin{tabular}{lc} 
Source of uncertainty & $\delta \mathcal{P}_{z} / \mathcal{P}_{z}$ \\
\hline Detector analysing power & $0.15-0.2 \%$ \\
Detector linearity & $0.1 \%$ \\
Laser polarisation & $0.1 \%$ \\
Electronic noise and beam jitter & $0.05 \%$ \\
\hline Total & $0.25 \%$
\end{tabular}

Table 6: Uncertainty budget for the Compton polarimeters.

All the contributions in table 6 are expected to be uncorrelated between all four polarimeters. Thus, additional precision could be gained from a cross calibration of the polarimeters in absence of collisions, if the spin transport from one location to the other is sufficiently well understood. In presence of collisions, both polarimeters fulfil very complementary tasks: While the downstream polarimeter in principle gives access to the depolarisation in collision, the upstream polarimeter provides a clean measurement of the initial polarisation and resolves possible time dependent patterns, e.g. inside a bunch train.

\section{Cross calibration of the Polarimeters}

In absence of collisions at the $e^{+} e^{-}$IP, the polarisation measurement at the upstream polarimeter can be propagated by spin tracking along the BDS and predict the expected

\footnotetext{
${ }^{3}$ I.e. that the relevant element of the transfer matrix between $e^{+} e^{-}$IP and the downstream polarimeter Compton IP is $\left|R_{22}\right|=0.5$.
} 
polarisation at the downstream polarimeter. Provided that the spin propagation effects are well under control, the two polarimeters can thus be cross calibrated. However, it is crucial to understand how deviations due to instrumental effects of the polarimeters can be disentangled from effects caused by the spin transport. Therefore, it is important to understand the individual impact of the various effects which could influence the spin transport between the polarimeters. Both the cross calibration of the polarimeters and the understanding of the spin transport are crucial to finally assess the collision effects and to extract the luminosity-weighted polarisation.

We studied the spin transport with STALC based on the SB2009_Nov10 lattice [41], which differs from the RDR lattice with respect to the new location of the upstream polarimeter ${ }^{4}$. For the positrons, the tracking starts at the beginning of the BDS (dashed line in figure 1). On the electron side, the simulation starts at the beginning of the positron production system, thus including the "dogleg" to bypass the positron source target area. In both cases, the beams are initialised according to the twiss parameters of the lattice and the TDR beam energy spreads. However, the final focus system of the SB2009_Nov10 lattice was not yet adapted to the TDR design and thus produces the RDR beta functions at the $e^{+} e^{-}$IP. In absence of collisions, no significant impact on the spin transport is expected due to this issue.

To avoid numerical problems, some magnets have been sliced into smaller units as described in chapter 7 of [24]. In each studied configuration, 1000 individual bunches have been tracked, each bunch with 40000 macroparticles 5 . To each macroparticle, a polarisation vector with a length of $|\overrightarrow{\mathcal{P}}|_{\max }=0.8$ is assigned. At the beginning of the tracking, the polarisation vectors are assumed to be oriented such that they are parallel to the orbit if the macroparticle trajectory is parallel to the orbit as well, and rotated analogously to T-BMT precession otherwise. In this case, the spin fan-out is expected to be described by equation 6. This spin configuration has already implicitly been assumed in earlier studies [25, 43]. The effects of a slightly different spin configuration will be examined in section 6 .

Figure 5 shows the polarisation $|\overrightarrow{\mathcal{P}}|$ and its longitudinal component $\mathcal{P}_{z}$ of the electron beam at the upstream polarimeter and between the $e^{+} e^{-}$IP and the downstream polarimeter for perfect alignment and in absence of detector magnets and crab cavities. It shows that the final focus magnets (in front of the IP) and the extraction line quadrupoles affect $|\overrightarrow{\mathcal{P}}|$ and $\mathcal{P}_{z}$ likewise, while the polarisation vector precesses in the dipole chicanes, but returns to a longitudinal configuration behind a pair of dipoles with opposite fields. The influence of the quadrupoles is well described by the spin fan-out prediction $f\left(\theta_{r}\right)$ (equation 6) which is drawn as green dash-dotted line. The residual differences stem most likely from the beam energy spread, which is not taken into account by $f\left(\theta_{r}\right)$, or from the betatron oscillations.

Table 7 lists selected bunch parameters from the simulation (and calculated from the design values, as far as possible) at the polarimeters and at the $e^{+} e^{-}$IP, where the latter serves mainly for illustration here, since it does not affect the cross calibration

\footnotetext{
${ }^{4}$ Due to its separation from the emittance measurement following earlier recommendations 42 .

${ }^{5}$ Thus, for a design bunch population of $N_{e}=2 \cdot 10^{10}$, one macroparticle represents $5 \cdot 10^{5}$ electrons.
} 


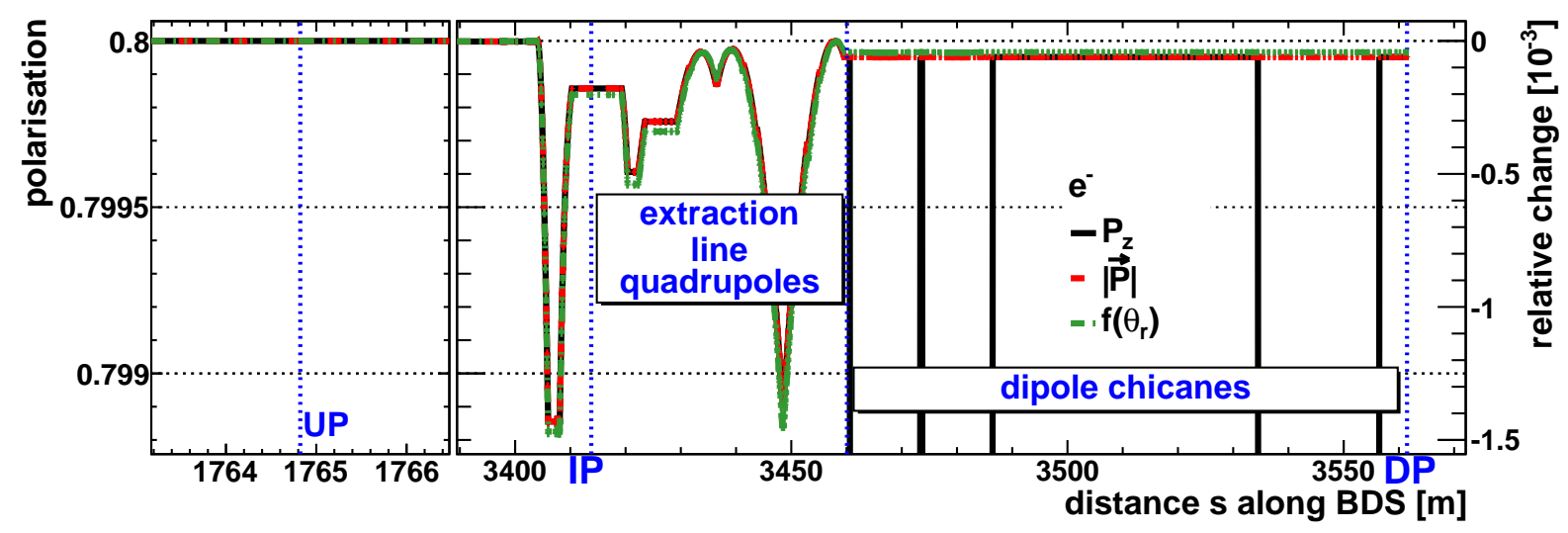

Figure 5: Longitudinal polarisation $\mathcal{P}_{z}$ (black, solid), magnitude of the polarisation vector $|\overrightarrow{\mathcal{P}}|$ (red, dashed) and the function $f\left(\theta_{r}\right)$ of the angular divergence defined in equation 6 (green, dash-dotted) of the electron beam at the upstream polarimeter (UP, left part) and between $e^{+} e^{-}$IP and downstream polarimeter (DP, right part).

\begin{tabular}{|c|c|c|c|c|c|}
\hline Simulation & & UP & $e^{+} e^{-} \mathrm{IP}$ & \multicolumn{2}{|c|}{ DP } \\
\hline$\theta_{r}$ & {$[\mu \mathrm{rad}]$} & $0.986 \pm 0.004$ & $35.4 \pm 0.1$ & \multicolumn{2}{|c|}{$16.27 \pm 0.06$} \\
\hline$\left(0.8-\mathcal{P}_{z}\right)$ & {$\left[10^{-6}\right]$} & $0.2 \pm \varepsilon$ & $143.0 \pm 0.8$ & \multicolumn{2}{|c|}{$48.4 \pm 0.3$} \\
\hline$(0.8-|\overrightarrow{\mathcal{P}}|)$ & {$\left[10^{-6}\right]$} & $0.2 \pm \varepsilon$ & $143.0 \pm 0.8$ & \multicolumn{2}{|c|}{$48.4 \pm 0.3$} \\
\hline \multirow[t]{2}{*}{$\left(0.8-f\left(\theta_{r}\right)\right)$} & {$\left[10^{-6}\right]$} & $0.1 \pm \varepsilon$ & $162 \pm 1$ & \multicolumn{2}{|c|}{$34.2 \pm 0.2$} \\
\hline & & & $e^{+} e^{-} \mathrm{IP}$ & \multicolumn{2}{|c|}{ DP } \\
\hline \multicolumn{3}{|l|}{ Design values } & RDR & RDR & TDR \\
\hline$\theta_{r}$ & {$[\mu \mathrm{rad}]$} & & 35 & 17 & 23 \\
\hline$\left(0.8-f\left(\theta_{r}\right)\right)$ & {$\left[10^{-3}\right]$} & & 0.157 & 0.039 & 0.064 \\
\hline
\end{tabular}

Table 7: Upper table: Angular divergence $\theta_{r}$, longitudinal polarisation $\mathcal{P}_{z}$, magnitude of the polarisation vector $|\overrightarrow{\mathcal{P}}|$ and the function $f\left(\theta_{r}\right)$ as defined in equation 6 of the electron beam at the polarimeters $\left(\mathrm{UP} / \mathrm{DP}=\right.$ up-/downstream polarimeter) and the $e^{+} e^{-} \mathrm{IP}$. Lower table: $\theta_{r}$ and $f\left(\theta_{r}\right)$ calculated from the design values (table 1). The accuracy of the simulation is estimated to be $10^{-7}$ at best. Therefore, uncertainties below $0.5 \cdot 10^{-7}$ are denoted as $\varepsilon$. Uncertainties between $0.5 \cdot 10^{-7}$ and $1 \cdot 10^{-7}$ are rounded up.

of the polarimeters. In the context of the envisaged precision of $0.1 \%$, the simulation results are in good agreement with the values predicted based on the angular divergence $\theta_{r}$. On this basis, the consequences of the stronger focussing for the TDR parameters can be estimated as well. Assuming the spin fan-out at the upstream polarimeter to be negligible, the relative difference in $\mathcal{P}_{z}$ at the two polarimeters due to spin fan-out for TDR parameters amounts to $\Delta \mathcal{P}_{z} / \mathcal{P}_{z}=8.0 \cdot 10^{-5}$. This is $64 \%$ larger than for RDR parameters, but still very small compared to the aim of $10^{-3}$.

We also studied the effect of a finite knowledge of the beam parameters, in particular the emittances. A variation of $10 \%$ leads to a negligible change in polarisation of $3 \cdot 10^{-5}$.

The effects arising from the emission of synchrotron radiation in the beamline magnets have been found to be negligible as well: The change in polarisation due to spin-flips 
between the two polarimeters has been calculated to be $<10^{-6}$. The energy loss due to emission of synchrotron radiation and the resulting changes in the particle trajectories change the polarisation by $5 \cdot 10^{-6}$. For more details, see section 9.1 of $[24]$.

\subsection{Beam Alignment at the Polarimeters and at the $e^{+} e^{-}$IP}

In order to avoid measuring values for $\mathcal{P}_{z}$ at the polarimeters and at the $e^{+} e^{-}$IP which differ a priori due to T-BMT precession, there should be no relative angle between the orbits at these points. Therefore, no effective bending angle between these points is foreseen in the BDS design.

Misalignments, however, might lead to relative incident angles $\Delta \vartheta_{\text {bunch }}$, which translates to a precession of the polarisation vector by an angle of $(1+a \gamma) \cdot \Delta \vartheta_{\text {bunch }}$ (equation 5) if the energy spread can be neglected [44]. The design requirement on the relative beam alignment between the polarimeter locations is $\Delta \vartheta_{\text {bunch }} \leq 50 \mu \mathrm{rad}$, driven by polarimetry demands. To achieve the maximum longitudinal polarisation, spin rotators in front of the main linac are used to adjust the polarisation vector parallel to the beam at the upstream polarimeter. This is expected to be possible with an uncertainty of $\Delta \vartheta_{\text {pol }}=25 \mathrm{mrad}{ }^{6}$.

For a beam energy of $250 \mathrm{GeV}$, these two contributions give the following total uncertainty on the polar angle of the polarisation vector:

$$
\Delta \vartheta_{\mathrm{pol}}^{\mathrm{tot}}=\sqrt{\Delta \vartheta_{\mathrm{pol}}^{2}+\left((1+a \gamma) \cdot \Delta \vartheta_{\mathrm{bunch}}\right)^{2}}=38 \mathrm{mrad}
$$

The corresponding uncertainty of the longitudinal polarisation amounts to

$$
\Delta \mathcal{P}_{z} / \mathcal{P}_{z}=1-\cos \left(\Delta \vartheta_{\text {pol }}^{\text {tot }}\right)=0.72 \cdot 10^{-3}
$$

The contribution from the incident angles increases with the beam energy. For a beam energy of $500 \mathrm{GeV}$ instead of $250 \mathrm{GeV}, \Delta \vartheta_{\text {pol }}^{\text {tot }}$ rises to $62 \mathrm{mrad}$ and $\Delta \mathcal{P}_{z} / \mathcal{P}_{z}$ to $1.9 \cdot 10^{-3}$. This is by far dominated by the contribution from the beam alignment: even for perfect alignment of the polarisation vector, $\Delta \mathcal{P}_{z} / \mathcal{P}_{z}$ would still amount to $1.6 \cdot 10^{-3}$. Thus, an upgrade to a collision energy of $1 \mathrm{TeV}$ would require an improved beam alignment in order to achieve the precision goal of $0.1 \%$ for the spin transport in the BDS.

A computational correction of the measured $\mathcal{P}_{z}$ for known incident angles would in principle be possible, but gives an additional contribution to the uncertainty of $\mathcal{P}_{z}$. As shown in section 7.4 of [24], such a correction seems extremely difficult in view of the precision goal of $0.1 \%$, since the contribution to $\Delta \mathcal{P}_{z} / \mathcal{P}_{z}$ grows with the angle itself as well as with its uncertainty. Therefore, the beam orbits at the polarimeters and at the $e^{+} e^{-}$IP have to be aligned by correction magnets. This correction method gives an additional contribution to the uncertainty of $\mathcal{P}_{z}$, which is discussed in the following.

\footnotetext{
${ }^{6} \mathrm{~A}$ possible scheme to measure the alignment of the polarisation vector is presented in 27 .
} 


\subsection{Residual Effects from the Beam Orbit Correction}

Misalignments of magnets as described in section 2.3 lead to misalignments of the beams. To keep the beams close to the design orbit and bring the bunches to collision at the $e^{+} e^{-}$ $\mathrm{IP}$, the BDS is equipped with a number of correction dipoles. However, these dipoles can not prevent the beam from leaving the design orbit in the first place, but only bend it back to the design orbit. While it was assumed in the previous section that a re-alignment of the beam orbit would also fully restore the alignment of the polarisation vector, the accuracy of this assumption will be tested in this section.

\begin{tabular}{ll} 
Sample name & Description \\
\hline no M & No misalignments (same data as for figure 5). \\
M5 & RMS size of all offsets $5 \mu \mathrm{m}$, RMS size of all rotations $5 \mu \mathrm{rad}$. \\
M10 & RMS size of all offsets $10 \mu \mathrm{m}$, RMS size of all rotations $10 \mu \mathrm{rad}$.
\end{tabular}

Table 8: Simulated samples for the investigation of the effects of magnet misalignments.

For this purpose, random misalignments of the beamline magnets, i.e. offsets and rotations in all three dimensions have been introduced into the simulation. Three data samples with different RMS sizes of misalignments have been produced as listed in table 8 . For each of the 1000 bunches per sample, new misalignments are generated. Correlations in space or time have not been taken into account for the misalignments. To correct the beam orbit, the corrector magnets foreseen in the lattice are used. The magnet strengths are computed from the beam positions at the beam position monitors (BPMs) [45] such that the beam position offsets measured by the BPMs are minimised. For the current study, negligible BPM resolutions have been assumed. At the $e^{+} e^{-}$IP, the fast-feedback correction is emulated which adjusts the beam position and incident angle according to the foreseen tolerances. The correction procedure is described in detail in 24.

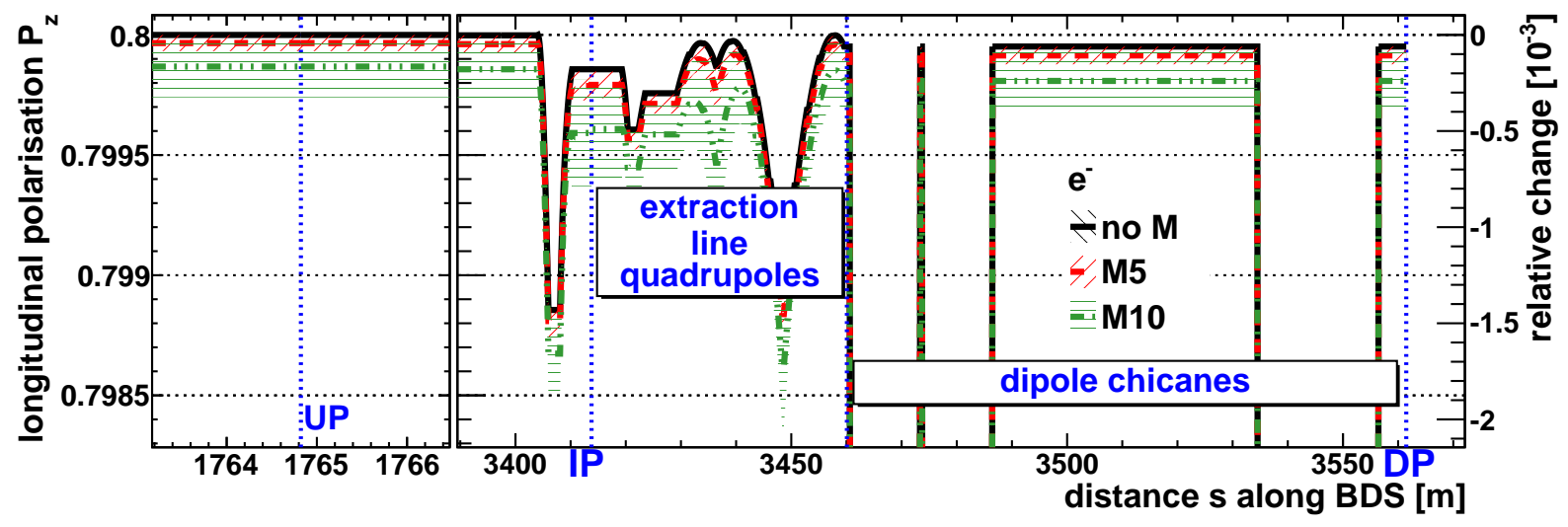

Figure 6: Longitudinal polarisation $\mathcal{P}_{z}$ of the electron beam at the upstream polarimeter (UP, left part) and between $e^{+} e^{-}$IP and downstream polarimeter (DP, right part) for the data samples listed in table 8. The uncertainty bands correspond to the RMS spread of all runs. For the perfectly aligned case, the band is too small to be visible.

Figure 6 shows the longitudinal polarisation $\mathcal{P}_{z}$ at the upstream polarimeter and between the $e^{+} e^{-}$IP for the same data samples. In addition, table 9 lists selected bunch 


\begin{tabular}{|c|c|c|c|c|c|}
\hline & \multicolumn{3}{|r|}{ no $M$} & M5 & M10 \\
\hline Upstream & $\vartheta_{\text {bunch }}$ & {$[\mu \mathrm{rad}]$} & $0.006 \pm 0.004$ & $2 \pm 1$ & $3 \pm 2$ \\
\hline polarimeter & $\theta_{r}$ & {$[\mu \mathrm{rad}]$} & $0.986 \pm 0.004$ & $0.989 \pm 0.005$ & $1.00 \pm 0.01$ \\
\hline & $\left(0.8-\mathcal{P}_{z}\right)$ & {$\left[10^{-6}\right]$} & $0.2 \pm \varepsilon$ & $34 \pm 34$ & $132 \pm 128$ \\
\hline & $(0.8-|\overrightarrow{\mathcal{P}}|)$ & {$\left[10^{-6}\right]$} & $0.2 \pm 0.1$ & $0.2 \pm \varepsilon$ & $0.3 \pm 0.1$ \\
\hline & $\left(0.8-f\left(\theta_{r}\right)\right)$ & {$\left[10^{-6}\right]$} & $0.1 \pm \varepsilon$ & $0.1 \pm \varepsilon$ & $0.1 \pm \varepsilon$ \\
\hline Downstream & $\vartheta_{\text {bunch }}$ & {$[\mu \mathrm{rad}]$} & $0.21 \pm 0.08$ & $0.7 \pm 0.4$ & $1.2 \pm 0.6$ \\
\hline polar & $\theta_{r}$ & {$[\mu \mathrm{rad}]$} & $16.27 \pm 0.06$ & $16.4 \pm 0.4$ & $16.7 \pm 0.9$ \\
\hline & $\left(0.8-\mathcal{P}_{z}\right)$ & {$\left[10^{-6}\right]$} & $48.4 \pm 0.3$ & $86 \pm 37$ & $192 \pm 138$ \\
\hline & $(0.8-|\overrightarrow{\mathcal{P}}|)$ & {$\left[10^{-6}\right]$} & $48.4 \pm 0.3$ & $49 \pm 2$ & $50 \pm 5$ \\
\hline & $\left(0.8-f\left(\theta_{r}\right)\right)$ & {$\left[10^{-6}\right]$} & $34.2 \pm 0.2$ & $35 \pm 2$ & $36 \pm 4$ \\
\hline
\end{tabular}

Table 9: Incident angle $\vartheta_{\text {bunch }}$, angular divergence $\theta_{r}$, longitudinal polarisation $\mathcal{P}_{z}$, magnitude of the polarisation vector $|\overrightarrow{\mathcal{P}}|$ and the function $f\left(\theta_{r}\right)$ as defined in equation 6 of the electron beam at the polarimeters for the data samples listed in table 8 . For $\varepsilon$, see the explanation in the caption of table 7

parameters of the electron beam at the polarimeters for different sizes of misalignments for the data samples listed in table 8 . The uncertainties in the table and the shaded areas in the figure denote the RMS spread of $\mathcal{P}_{z}$ of the simulated bunches. These spreads reflect the possible variations depending on the exact misalignments of the individual magnets, which lead to different trajectories for each bunch.

While the norm of the polarisation vector is hardly affected by the misalignments, there is a larger effect on the longitudinal component $\mathcal{P}_{z}$. The values of $|\overrightarrow{\mathcal{P}}|$ remain consistent with $f\left(\theta_{r}\right)$ (cf. section 3.1); thus, the behaviour of $|\overrightarrow{\mathcal{P}}|$ is still fully explained by spin fan-out. The increases in the mean value and the uncertainty of $\vartheta_{\text {bunch }}$ do, however, not suffice to explain the decrease in $\mathcal{P}_{z}$ by precession of the polarisation vector according to equation 5: $\Delta \mathcal{P}_{z}=2 \cdot 10^{-4}$ would correspond to $\vartheta_{\text {bunch }}=39 \mu \mathrm{rad}$, while $\vartheta_{\text {bunch }}=5 \mu \mathrm{rad}$ would yield only $\Delta \mathcal{P}_{z}=4 \cdot 10^{-6}$. This discrepancy appears after one of the correction magnets in the "dogleg" where the electron beam by-passes the photon target area of the positron source (cf. fig. 1), possibly due to non-commutation of rotations about different axes: while all deflections of the bunch by misaligned magnets are compensated for with the corresponding deflections at the correction magnets, this compensation does not work perfectly for the polarisation vector which performs rotations by the $(1+a \gamma)$-fold deflection angles.

In case of purely static misalignments, the decisive quantity for the cross calibration of the polarimeters is the residual difference of the longitudinal polarisation at both locations. Figure 7 shows this difference in terms of $\Delta \mathcal{P}_{z}(\mathrm{UP}, \mathrm{DP}):=\left(\mathcal{P}_{z}^{\mathrm{UP}}-\mathcal{P}_{z}^{\mathrm{DP}}\right) / \sqrt{2}$ for the sample "M10". It follows an approximately Gaussian distribution with an RMS spread of $0.04 \cdot 10^{-3}$. This is about a factor 10 smaller than the variations of $\mathcal{P}_{z}$ at any individual location, i.e. the uncertainty band in figure 6. Nevertheless, we conservatively estimate the uncertainty from the magnet misalignments and the beam orbit correction based on figure 6, in order to allow for time-dependent misalignments which have not 


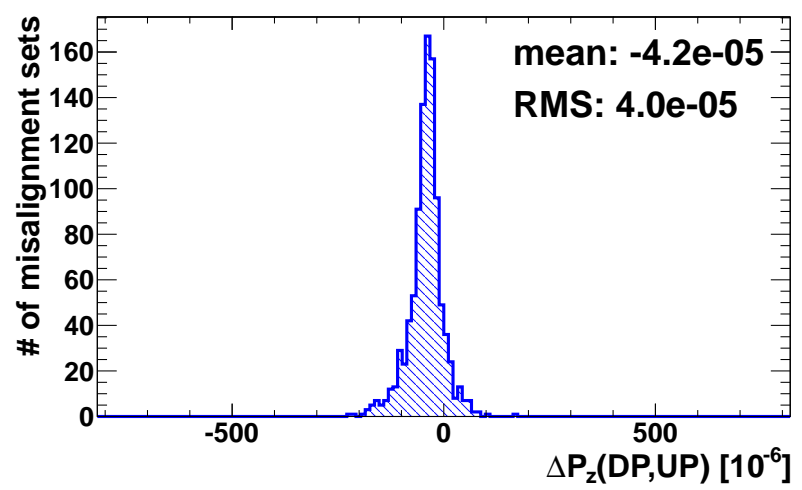

Figure 7: Difference of the longitudinal polarisation $\mathcal{P}_{z}$ of the electron beam at the upstream polarimeter (UP) and the downstream polarimeter (DP) for the misalignment sample "M10".

been explicitely studied here. Thus, we define this uncertainty as the relative deviation of the lower bound of the spread for $\mathcal{P}_{z}$ for the samples with misalignments from the mean value of $\mathcal{P}_{z}$ for the sample without misalignments (both values at the downstream polarimeter, neglecting the decrease in $\mathcal{P}_{z}$ at the upstream polarimeter). This yields a relative uncertainty of $0.09 \cdot 10^{-3}$ for the sample "M5" and $0.35 \cdot 10^{-3}$ for the sample "M10".

Thus, we conclude that misalignments of beamline elements are not the dominating source of systematic uncertainty, but still can give a sizable contribution. Especially the impact of time-dependent effects should be studied in more detail based on realistic ground-motion models of the selected ILC site, also taking into account finite BPM resolutions.

\subsection{Detector Magnets and Crab Cavities}

In this section we investigate more closely the interaction region devices and their impact on the spin transport. When operating the machine without collisions, the detector magnets and the crab cavities could be switched on or off. Thus, their impact on the polarisation measured at the downstream polarimeter could rather easily be disentangled from instrumental differences between the up- and downstream polarimeter measurements.

\subsubsection{Detector Magnets}

The beams enter the detector under an angle of $7 \mathrm{mrad}$, which is half the beam-beam crossing angle. Hence, both the main solenoid and the anti-DID feature magnetic field components parallel and perpendicular to the beam.

The parallel field components might destroy the synchronisation between the angles of the beam orbit and the polarisation vector, such that $\mathcal{P}_{z}$ takes different values at the $e^{+} e^{-}$IP and at the polarimeters even if the beam orbit is aligned parallel at these three 
locations. This effect has, however, turned out to be negligible $\left(\Delta \mathcal{P}_{z} \sim 10^{-5}\right)[24 \mid$ despite the strong longitudinal field of the solenoid. This can be understood since the transverse components of the polarisation vector, which would undergo the precession about the longitudinal axis, are rather small, and since the precession about the longitudinal axis does not speed up with rising particle energies (cf. equation 2). The effect of the longitudinal field components would have to be re-evaluated for physics operation of the ILC with transverse beam polarisation.

The perpendicular field components act like dipole magnets on the beam. The resulting deflection would alter the beam position by several micrometers at the $e^{+} e^{-} \mathrm{IP}$, such that no collisions would occur, and several millimeters at the downstream polarimeter. To correct the beam position, additional dipole magnets in front of and behind the detector are foreseen, which are not contained in the lattice files [41] yet. For establishing luminosity, only the relative angles of the beams to each other are relevant [46]. However, polarimetry requires also the angles of the beam orbits at the polarimeters to be aligned parallel to the beam orbits at the $e^{+} e^{-}$IP to a level of $50 \mu \mathrm{rad}$ (cf. section 5.1) in presence of collisions. Without any beam orbit correction, the fields of the detector magnets would induce a deflection angle of $\vartheta_{\text {bunch }}=185 \mu \mathrm{rad}$ at the $e^{+} e^{-} \mathrm{IP}$, which leads to a decrease in longitudinal polarisation by $\Delta \mathcal{P}_{z}=4.5 \cdot 10^{-3}$ due to T-BMT precession [24]. As explained in the previous section, such a deflection angle at the $e^{+} e^{-}$IP can only be corrected for if it can be measured to significantly better than $10 \%$. If for instance the above $\vartheta_{\text {bunch }}=185 \mu \mathrm{rad}$ are known to $15 \mu \mathrm{rad}(8 \%)$, the resulting uncertainty on the polarisation from this correction alone amounts to $0.1 \%$. Thus, it is very important to foresee sufficient orbit correction possibilities such that not only the relative, but also the absolute angles of the beams at the $e^{+} e^{-}$IP can be adjusted in presence of the detector magnets.

\subsubsection{Crab Cavities}

To initiate the rotation of the particle bunches, the crab cavities generate a time-dependent electromagnetic field. In the centre of the cavity and at the time of the bunch passages, this field can be approximated by a time-dependent magnetic dipole field, which deflects the bunch particles horizontally by an angle proportional to the longitudinal distance from the bunch centre. This deflection goes along with the corresponding spin precession (equation 5), which results in a spin fan-out along the $z$-axis and thus in a decrease of the polarisation. The effect on the polarisation can, however, be expected to be small, since the fan-out vanishes at the bunch centre, where the majority of the particles is located. Simulations have shown that the effect on the polarisation is smaller than $10^{-5}$ (section 8.1 in $[24]$ ).

\subsection{Cross Calibration of the Polarimeters}

Table 10 summarises the uncertainties on the spin transport between the polarimeters for the cross calibration in absence of collisions and for a beam energy of $250 \mathrm{GeV}$. The major contribution comes from the alignment precision of the beam and the polarisation 
vector (section 5.1). A second large contribution comes from the beam orbit correction (section 5.2), while all other contributions listed are negligible. All contributions sum up to an uncertainty of $0.080 \%$, which matches the goal of at most $0.1 \%$. For higher beam energies, many of the contributions can be expected to increase. The contribution from the alignment precision alone rises to $1.9 \cdot 10^{-3}$ for a beam energy of $500 \mathrm{GeV}$, which necessitates a better beam alignment.

\begin{tabular}{lr} 
Contribution & $\delta \mathcal{P}_{z} / \mathcal{P}_{z}\left[10^{-3}\right]$ \\
\hline Beam and polarisation alignment at polarimeters & 0.72 \\
(assuming $\left.\Delta \vartheta_{\text {bunch }}=50 \mu \mathrm{rad}, \Delta \vartheta_{\text {pol }}=25 \mathrm{mrad}\right)$ & 0.35 \\
Random misalignments $(10 \mu \mathrm{m} / \mu \mathrm{rad})$ with beam orbit correction & 0.03 \\
Variation in beam parameters $(10 \%$ in the emittances) & 0.01 \\
Longitudinal precession in detector magnets & $<0.01$ \\
Bunch rotation to compensate the beam crossing angle & 0.005 \\
Emission of synchrotron radiation & 0.80
\end{tabular}

Table 10: Contributions to the uncertainty of the spin transport from the upstream to the downstream polarimeter for a beam energy of $250 \mathrm{GeV}$ in the absence of collisions.

\section{Luminosity-Weighted Average Polarisation}

In this section, we discuss the relevant quantity for the interpretation of collision data, i.e. the luminosity-weighted average polarisation at the IP. We studied the direct depolarisation in collisions as well as for the first time the effects which arise at the downstream polarimeter location and their interplay with polarimetry. Finally, we lay out a strategy how to access the luminosity-weighted average polarisation from polarimeter measurements and how to compare this to collision data.

In addition to the RDR beam parameter set used in section 5, also collisions with TDR beam parameters have been simulated (cf. section 2.1). As explained in section 5, the current lattice features a final focus according to RDR parameters at the $e^{+} e^{-} \mathrm{IP}$. However, no significant changes in the spin tracking upstream of the $e^{+} e^{-}$IP are expected. On the other hand, the extraction line lattice is suitable for both parameter sets. Thus, the beams are generated at the $e^{+} e^{-}$IP, passed to the simulation of the collisions, and then propagated to the downstream polarimeter.

These two data samples named "RDR" and "TDR" are both generated with the spin configuration as described in the beginning of section 5. This is well motivated since the spin transport is dominated by spin fan-out as shown in the previous section. To illustrate nevertheless the effects of a different initial spin configuration, a third sample "TDR*" has been generated: all macroparticle polarisation vectors are aligned along the beam axis at the $e^{+} e^{-}$IP, instead of being fanned out according to the focussing of the beam. Such a different spin configuration could e.g. be the result of the betatron oscillations (non-commutating rotations, cf. section 5.2. 
To be able to distinguish the contributions from spin precession and from energy losses, each of the three data samples has been resimulated with the particle energies fixed to $E=250 \mathrm{GeV}\left(\mathrm{RDR}_{0}, \mathrm{TDR}_{0}, \mathrm{TDR}_{0}^{*}\right)$. In other words, the bunches are generated without initial energy spread, and no energy loss due to synchrotron radiation or beamstrahlung (and thus no depolarisation due to Sokolov-Ternov effects) is simulated, so that spin precession is the only source of changes in polarisation. The definitions of all samples are summarised in table 11 .

\begin{tabular}{ll} 
Sample name & Description \\
\hline RDR & Beam parameters according to the RDR (cf. table 1), but with \\
& the energy spread according to the TDR (same parameters as \\
& in section 5). \\
& Beam parameters according to the TDR (cf. table 1). \\
TDR & Like TDR, but with a different initial spin configuration: all \\
TDR & macroparticle polarisation vectors are aligned along the $z$-axis \\
& at the IP, instead of being fanned out according to the focussing \\
& of the beam. \\
& Samples like above, but all particles with fixed energy $E=$ \\
& $250 \mathrm{GeV}$ (no energy spread, no synchrotron radiation or beam- \\
& strahlung).
\end{tabular}

Table 11: Simulated samples for the investigation of the beam-beam collision effects.

The luminosities of the simulated collisions amount to $(2.02 \pm 0.02) \cdot 10^{34} \mathrm{~cm}^{-2} \mathrm{~s}^{-1}$ and $(1.52 \pm 0.03) \cdot 10^{34} \mathrm{~cm}^{-2} \mathrm{~s}^{-1}$ for the samples RDR and TDR, respectively, which is in good agreement with the design luminosities (cf. table 1 ).

\subsection{Beam Properties at Downstream Polarimeter}

In absence of collisions, the bunches at the Compton IPs of the polarimeters are smaller than the laser spots or of similar size. In collision mode, the beams get disrupted by the beam-beam interaction. Although the downstream polarimeter is placed at a secondary focus of the extraction line optics, the refocussing is hampered by the larger emittance and the larger energy spread of the disrupted beam after the collision.

This is illustrated by figure 8, which shows the horizontal and vertical particle positions vs. the particle energies for the sample TDR at the downstream polarimeter without collisions (a,b) and after a collision (c-f). Since the downstream polarimeter is located in a vertical magnet chicane (figure 2), dispersion also contributes to the vertical beam size. The expected offset due to dispersion is indicated as a black line for comparison. In absence of collisions, the particle bunch sizes are well matched by the design laser spot size of $\sigma_{x \gamma}=\sigma_{y \gamma}=100 \mu \mathrm{m}$ (cf. section 4). In contrast, the disrupted beam after a collision extends over centimeters, although a large fraction of particles has lost no or only little energy and is still well focussed. These particles are confined to a spot of $\sim 100 \mu \mathrm{m}$ size, while the particles which have lost more energy are spread out much further and are not covered by the laser spot of the polarimeter. 


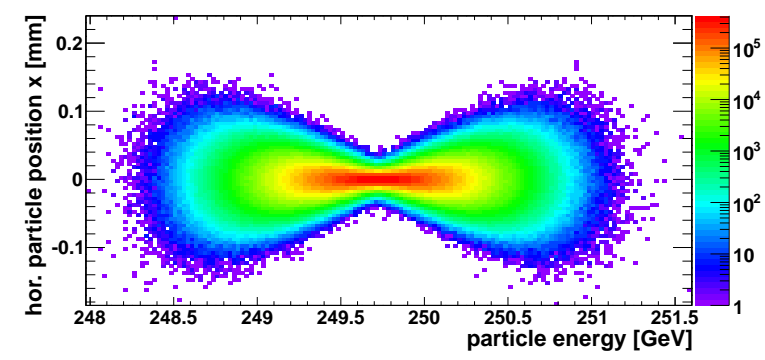

(a)

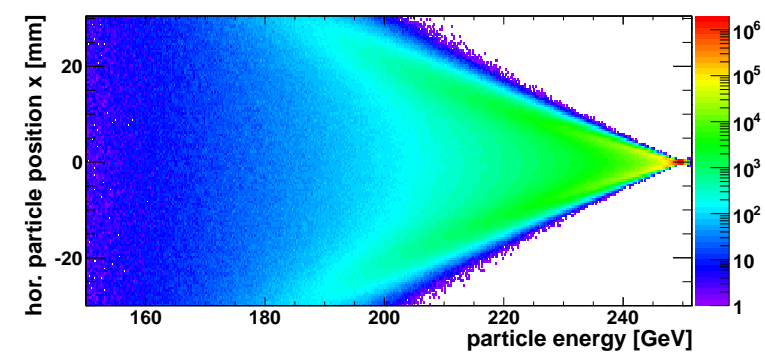

(c)

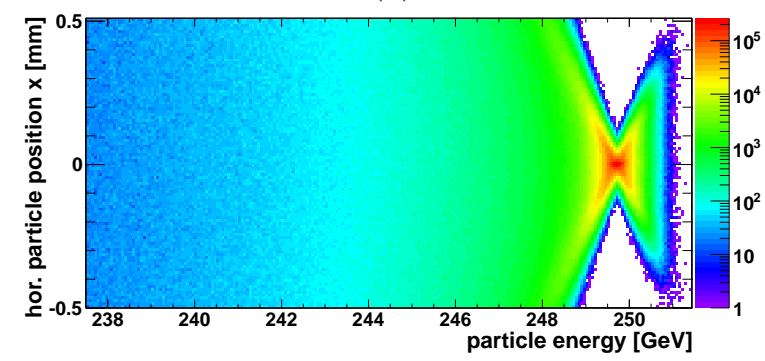

(e)

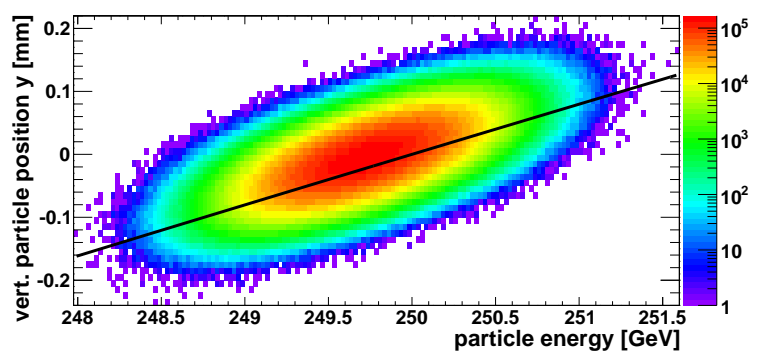

(b)

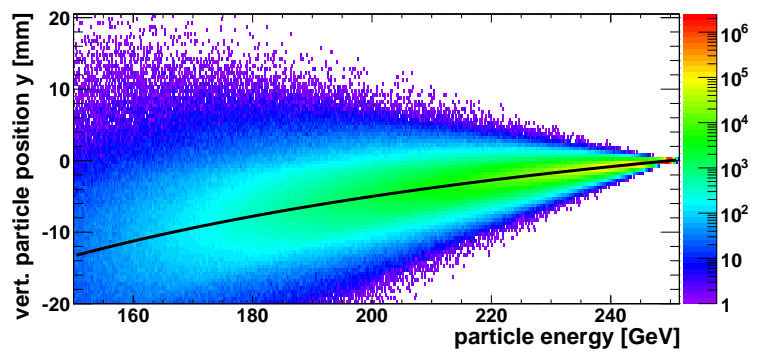

(d)

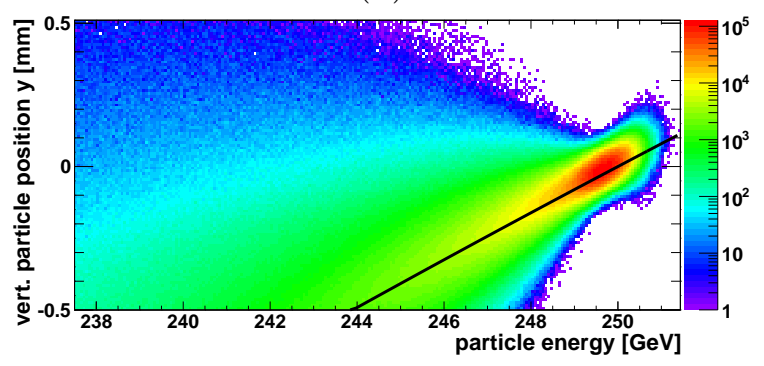

(f)

Figure 8: Horizontal and vertical particle positions vs the energy at the downstream polarimeter for 1000 electron bunches without collisions (a,b), after (c,d) the collision. $(e, f)$ show zooms into $(c, d)$. The black solid curve in $(b, d, f)$ shows the dispersion relation between $y$-position and energy.

As illustrated by figure 9, the longitudinal polarisation in the electron beam (sample TDR, after collision) correlates strongly with the position (a-c) and the energy (d) of the macroparticles, since particles which have been deflected more strongly by electromagnetic fields during the collision experience more spin precession and emit more beamstrahlung.

For a measurement using a laser spot of $\sim 100 \mu \mathrm{m}$ size, this implies that the measurement will not only be rather sensitive to the correct positioning of the laser, but also that the outcome of the measurement depends on the exact size of the laser spot at the Compton IP. As a first estimate of the effect on the downstream polarisation measurement, we investigated the measurable polarisation, which we define as the average longitudinal polarisation of the subset of macroparticles which would be hit by the laser, i.e. within a slice through the bunch defined by the laser spot size and the vertical crossing angle. 


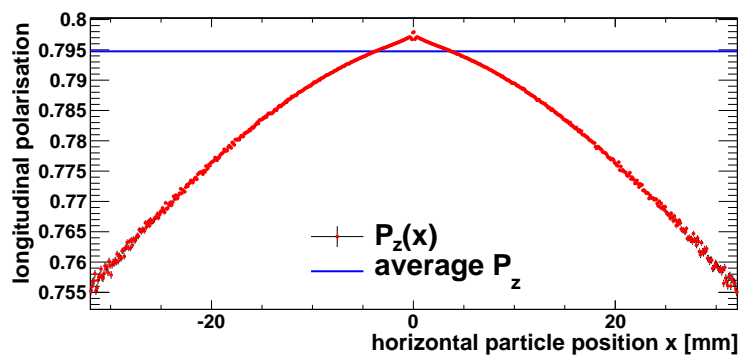

(a)

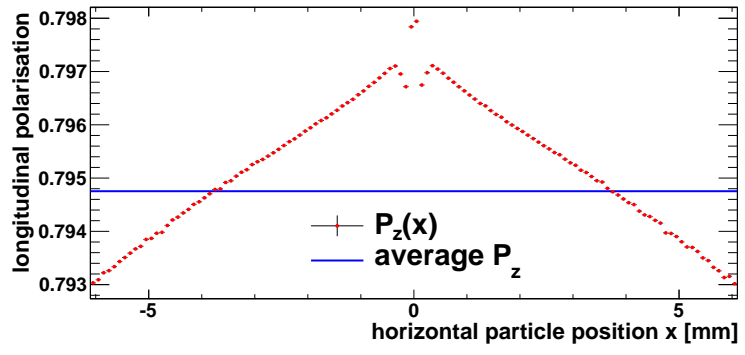

(c)

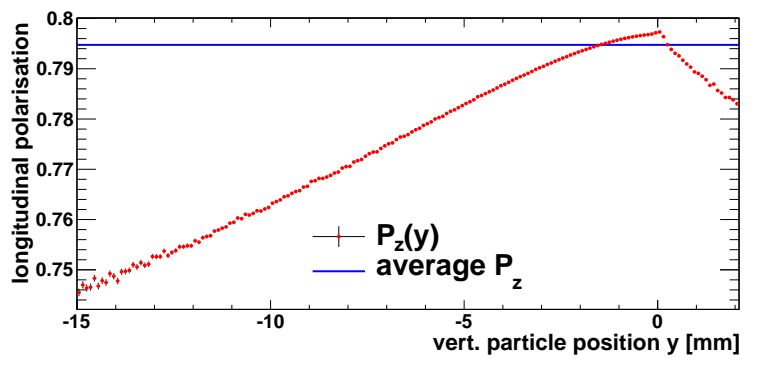

(b)

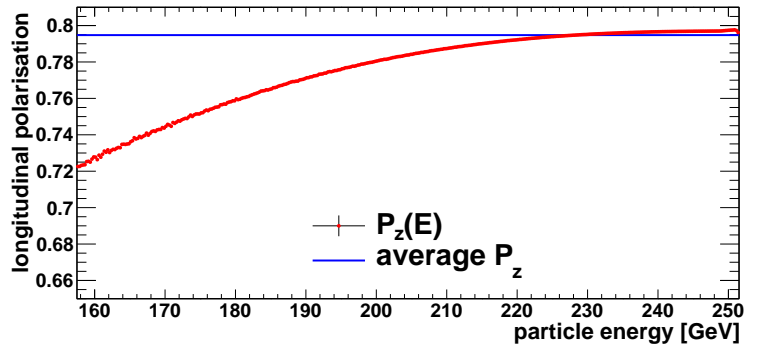

(d)

Figure 9: Longitudinal polarisation of the macroparticles versus the horizontal particle position $x$ (a) (and zoom (c)), the verticle particle position $y$ (b) and the particle energy $E(\mathrm{~d})$ at the downstream polarimeter.

\subsection{Collision Effects on Polarisation and Polarimetry}

As discussed in section 3.2, the luminosity-weighted average polarisation during collisions can be restored at the location of the downstream polarimeter if the extraction line quadrupoles halve the divergence angle compared to its value at the $e^{+} e^{-}$IP. This behaviour, however, is only obtained if spin fan-out is the dominating effect on the polarisation, which should be the case for the samples with pure T-BMT precession, $\mathrm{RDR}_{0}$, $\mathrm{TDR}_{0}$ and $\mathrm{TDR}_{0}^{*}$.

Figure 10 shows the polarisation $|\overrightarrow{\mathcal{P}}|$ of the electron beam between the IP and the downstream polarimeter for the case of $\mathrm{RDR}_{0}$ after the collision and without collisions. Due to the disruption in the collision, the divergence angle of the beam is enlarged from $33 \mu \mathrm{rad}$ to $205 \mu \mathrm{rad}$ at the IP, which leads to a decrease in polarisation of $0.5 \%$ by spin fan-out. The luminosity-weighted polarisation in the collision, indicated by the red dot at the IP, is lower than the incoming polarisation by $0.27 \%$, which is in accordance with equation 8. Behind the IP, the full tracking result is in perfect agreement with the expectation from pure spin precession, $f\left(\theta_{r}\right)$. At the location of the downstream polarimeter, the divergence angle is halved to $102 \mu \mathrm{rad}$ as desired. Thus, the value of the luminosity-weighted polarisation is reproduced at the downstream polarimeter to a level of $0.01 \%$ despite spin fan-out of several percent along the way. This larger spin fan-out occurs since the disrupted beam is more divergent and hence refocussed more strongly than a non-colliding beam. In summary, the design concept for the spin transport in the extraction line works perfectly if only T-BMT precession is taken into account, but no energy losses or radiative depolarisation. 


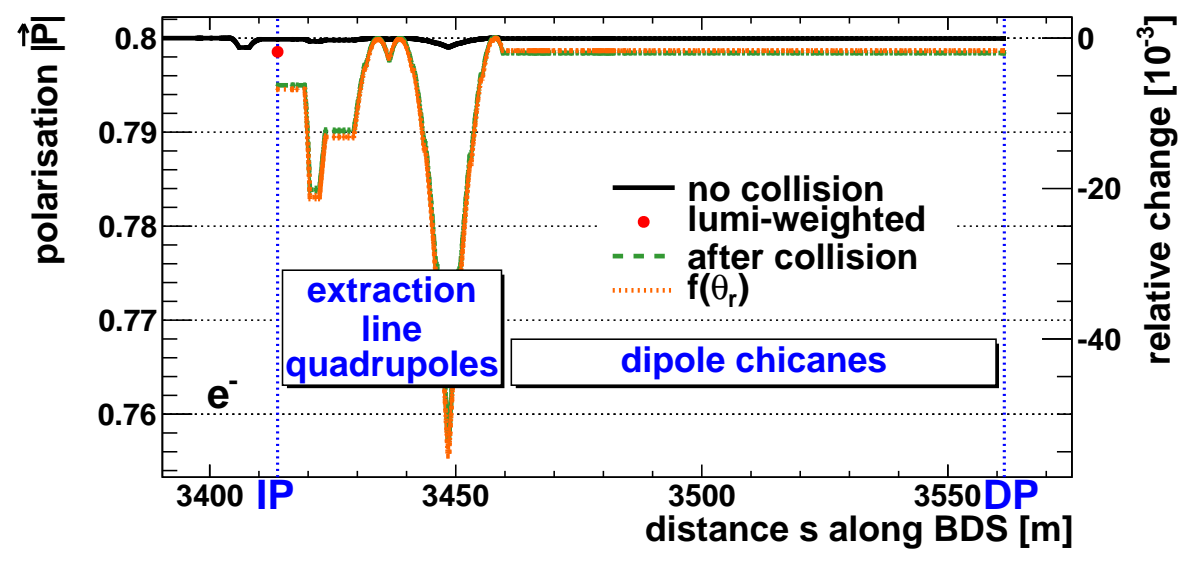

Figure 10: Magnitude of the polarisation vector of the electron beam along the BDS in the absence of any energy loss (data sample $\mathrm{RDR}_{0}$ ). Shown are the propagation in the case of no collisions, the different propagation after the collision, the luminosity-weighted polarisation in the collision and $f\left(\theta_{r}\right)$ after the collision.

In reality, however, energy loss and radiative depolarisation are non-negligible and need to be included in any realistic study. Therefore, all six cases have been simulated in analogy to figure 10, and the results are summarised in figure 11, by displaying the obtained polarisation values only at the points of interest. For the longitudinal polarisation, the measurable polarisation is indicated in addition for four different assumptions on the laser spot size $\sigma_{x \gamma}\left(=\sigma_{y \gamma}\right)$, always assuming a perfect centering of laser and electron beam.

The first column, $\mathrm{RDR}_{0}$, corresponds directly to figure 10 , showing again the perfect recovery of the luminosity-weighted average polarisation at the downstream polarimeter. Since the electron beam spot is fully hit by the laser, the measurable polarisation is identical to the longitudinal polarisation of the full bunch.

The next two columns show the results for TDR parameters, still for T-BMT precession only, but two different start configurations of the spins. Interestingly enough, the luminosity-weighted average polarisation and the polarisation at the downstream polarimeter start to deviate by $0.06-0.15 \%$ even without energy loss, both in magnitude and in longitudinal component. This could be due to the larger horizontal disruption parameter of the TDR parameter set $\left(D_{x}=0.3\right.$ compared to $D_{x}=0.17$ in the RDR case), since equation 8 assumes the horizontal disruption parameter to be small $\left(D_{x} \ll 1\right)$. Moreover, the size of this deviation depends on the initial spin configuration. This indicates that a full start-to-end spin tracking simulation from the electron and positron sources is highly desirable for the future.

The realistic results including energy loss and Sokolov-Ternov depolarisation are shown in the remainder of the figure. Compared to the corresponding sample without energy loss, the depolarisation in collisions increases in all three cases as expected. More importantly, also the differences between the polarisation at the downstream polarimeter and the luminosity-weighted average one increase to tolerable $0.07 \%$ in the RDR case, while it amounts to $0.17-0.24 \%$ in the TDR case, which exceeds the aim of $0.1 \%$. 


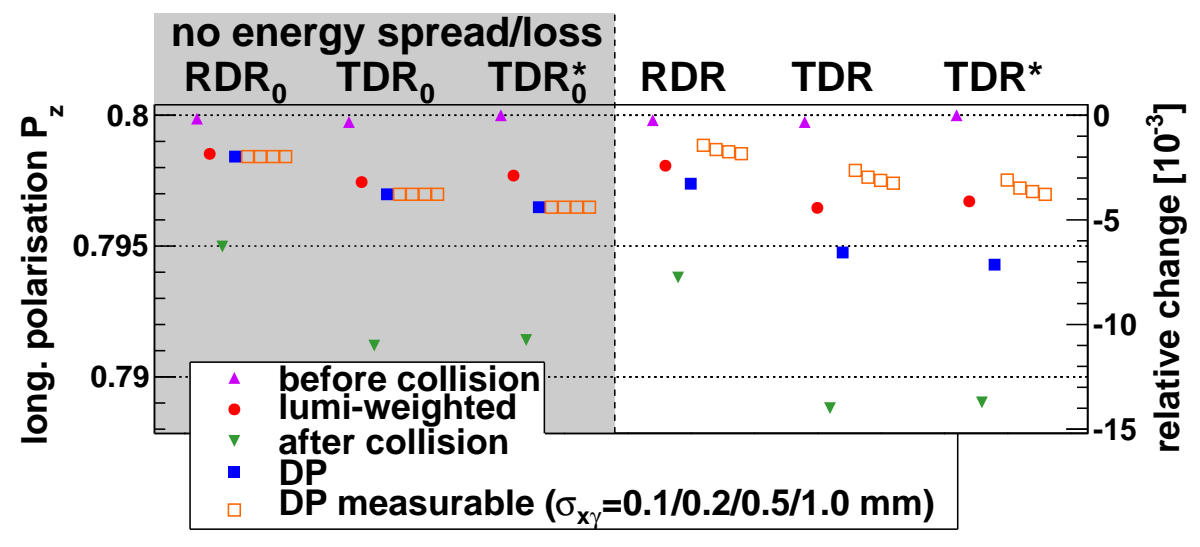

(a)

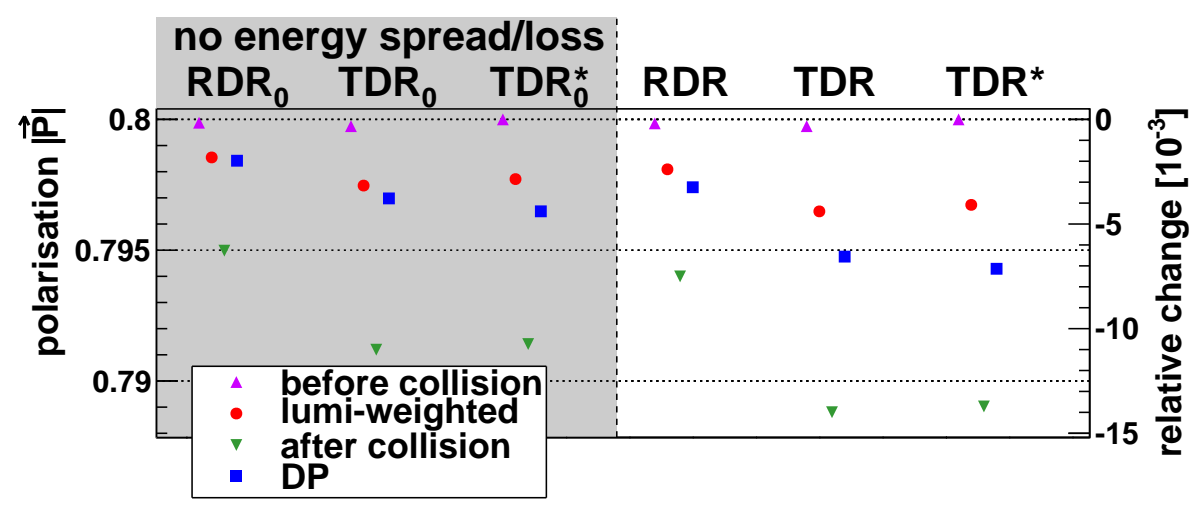

(b)

Figure 11: Electron beam polarisation along the BDS at the IP and at the polarimeters. Shown are the longitudinal polarisation $\mathcal{P}_{z}$ (a) and the magnitude of the polarisation vector $|\overrightarrow{\mathcal{P}}|$ (b) of the electron beam at the IP before and after the collision as well as the luminosity-weighted values and the values at the downstream polarimeter (DP). In (a), also the measurable longitudinal polarisation for different laser spot sizes $\sigma_{x \gamma}\left(=\sigma_{y \gamma}\right)$ is shown.

However, most strikingly the energy loss limits the refocussing of the beams, resulting in significantly increased beam sizes at the location of the downstream polarimeter discussed in the previous section. Now, the measurable polarisation deviates significantly from the total average at the downstream polarimeter by up to $0.3 \%$, and changes with the assumed size of the laser spot by up to $0.07 \%$. The difference between the measureable polarisation and the luminosity-weighted average at the IP is somewhat smaller due to a partial cancellation. In particular in the nominal TDR case, the net difference amounts to $0.15 \%$ for a nominal laser spot size of $100 \mu \mathrm{m}$. This number depends significantly on the actual beam parameters at the IP: for the RDR case, the analoguous difference is only $0.08 \%$.

For the positron beam, a different behaviour could be expected due to the different degree of polarisation, the different initial beam energy spread and the absence of the 
positron production system, which only exists in the electron beamline. However, the most dominant effect, T-BMT precession, scales linearly with $|\overrightarrow{\mathcal{P}}|$. Thus, the relative changes in the (longitudinal) polarisation of the positron beam differ only slightly from those of the electron beam 24.

Not yet included in the above numbers are additional contributions from non-perfect centering of the laser beam onto the electron beam nor any effect of the energy and position distributions of the incoming electrons on the analysing power of the downstream polarimeter, which needs to be addressed in future studies. The luminosity-weighted average polarisations above are expressed in the laboratory system, already accounting for the crossing angle of the beamlines, but not boosted to the centre-of-mass system.

\subsection{Accessing the Luminosity-Weighted Average Polarisation}

In view of the above results, assuming the downstream polarimeter to directly measure the luminosity-weighted average polarisation at the electron-positron interaction point $\mathcal{P}_{z}^{\text {lumi }}$ seems sufficient for percent-level, but not for permille-level precision. Instead, a more sophisticated strategy based on a detailed understanding of the collisions as a function of time is suggested in the following.

1. Cross-calibration of the polarimeters: The very first step, to be repeated regularly, e.g. during maintainance of the main detectors, is the cross-calibration of the polarimeters without collisions. The beam time requirement is given by the downstream polarimeter, which reaches a statistical precision of below $0.1 \%$ after about one hour 7. Systematic effects on the spin transport are expected to be $<0.1 \%$, provided the orbit alignment goals at the two polarimeter locations can be reached.

2. Upstream polarimeter: During collisions, the upstream polarimeter is essential to determine the initial polarisation value, which is expected to be $0.25 \%$ (RDR) to $0.4 \%$ (TDR) above $\mathcal{P}_{z}^{\text {lumi }}$, and to track time variations. Both aspects are needed to predict the expectation at the downstream polarimeter, which measures just one (or a few) bunches out of each train.

3. Knowledge of collision properties: In order to predict a) the depolarisation in collisions and b) the beam properties at the downstream polarimeter, all possible means to monitor the beam parameters before, during, and after collisions should be employed. This includes the energy spectrometers [13], the monitoring of the instantaneous luminosity, the beamstrahlung and the pair background in the forward calorimeters of the main detectors [47] and the Gamma Calorimeter in the downstream polarimeter chicane. From their combined information, the beam parameters, in particular $\sigma_{x e}$ and $\sigma_{y e}$ can be determined to about $10 \%$ [48. For comparison, the main difference between the RDR and TDR beam parameter sets studied here is a reduction of $\sigma_{x e}$ by $25 \%$. Thus we estimate that the depolarisation in collisions could be predicted to about $0.1 \%$ based on the measured beam parameters.

\footnotetext{
${ }^{7}$ It should be investigated in the future whether in absence of collisions the downstream measurement could collect sufficient statistics on similar timescales as the upstream polarimeter.
} 
4. Collision simulation: Based on theoretical understanding of the intense-field QED environment of the collisions, the knowledge of initial beam parameters and the post-

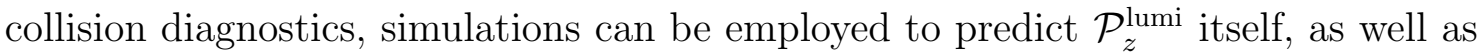
the energy and position distributions and the polarisation of the spent beam at the downstream polarimeter location. Redundancy in instrumentation is a key to validate the simulation tools.

5. Downstream polarimeter: The prediction of the beam properties at the downstream polarimeter location then needs to be employed to determine the changes in analysing power compared to the well defined situation without collisions. Furthermore, the effect of uncertainties in the laser alignment and focussing need to be assessed. The possibility to defocus the laser on purpose and/or scan the outer regions of the electron beam should be considered in order to "map out" the spent beam and verify the effects predicted by simulations.

6. Comparison of polarimeters and simulations: Based on the previous steps, the measured polarisation value at the downstream polarimeter can be extrapolated to the luminosity-weighted average at the IP. This needs to happen in a time-dependent manner, since the intensities of the bunch-bunch collisions might change during a bunch train and on longer time scales. This value can then be compared to the prediction for $\mathcal{P}_{z}^{\text {lumi }}$ based on the upstream polarimeter measurement and the beam parameter estimates. Only if sufficient agreement is found in this step, permillelevel accuracy on $\mathcal{P}_{z}^{\text {lumi }}$ can be claimed. It should be noted that once agreement is established, the extrapolated upstream polarimeter measurement will be the main "working horse" due to its ability to simultanously measure the polarisation for each bunch position in a train, while for the downstream polarimeter it takes order of days to sample each bunch position with permille-level statistical precision.

7. Polarisation values for physics analyses: The relevant effective polarisation for the physics process under study (cf. section 3.2) then needs to be calculated from values of $\mathcal{P}_{z}^{\text {lumi }}$ obtained for both beams from the beam-parameter-dependent extrapolation of the polarimeter measurements. Finally a luminosity-weighted average of the effective polarisations can be formed for each particular data set, including e.g. the analysis-specific run selection based on sub-detector availabilities. Here it should be noted that instrumental uncertainties of the polarimeters as well as effects of misalignments should be uncorrelated between both beams, while in particular the collision effects are due to the mutual influence of the beams on each other and thus are correlated to a large extent.

8. Comparison with collision data: After accumulation of a significant amount of collision data, the long-term average of $\mathcal{P}_{z}^{\text {lumi }}$ can be extracted also from the collision data themselves. Typically several $100 \mathrm{fb}^{-1}$ distributed over all four helicity configurations are needed for sub-percent statistical precision [6 8], where again time-dependencies and correlations can only be resolved based on polarimeter information. Nevertheless, polarisation-sensitive Standard Model processes with sufficiently large cross sections will provide an essential verification of the absolute polarisation scale. 


\section{Conclusions}

The polarimetery concept for the ILC is based on the combination of two complementary Laser-Compton polarimeters per beam and the long-term average polarisation determined from collision data. These three ingredients can only be exploited coherently in conjunction with detailed simulations of the spin transport and the beam-beam collision effects. For this purpose, the simulation framework STALC has been developed. It is not intrinsically limited to the ILC, but can be run on any lattice and beam parameter set.

In absence of collisions, various effects which could influence the spin transport between the polarimeters and, thus, e.g. their cross-calibration have been evaluated. The dominating uncertainty stems from the relative beam alignment at the two polarimeter locations. Here, the design goal of $\Delta \vartheta_{\text {bunch }} \leq 50 \mu \mathrm{rad}$ is just sufficient for permille-level precision for a beam energy of $250 \mathrm{GeV}$, while for the upgrade to $\sqrt{s}=1 \mathrm{TeV}$ an improvement to about $\Delta \vartheta_{\text {bunch }} \sim 25 \mu \mathrm{rad}$ would be required in order to maintain the same level of precision on the polarisation.

In presence of collisions, additional effects have to be considered with respect to the polarisation. This includes both the depolarisation in collision and thus the luminosityweighted average polarisation during the collision as well as the properties of the spent beam which influence the downstream polarimeter measurement. It has been shown that reducing the angular divergence at the downstream polarimeter with respect to the IP by a factor of two can only partially restore the luminosity-weighted average polarisation at the downstream polarimeter location, since for ILC beam parameters the energy loss and Sokolov-Ternov spin flips due to the emission of beamstrahlung cannot be fully neglected anymore. The influence of the spent beam properties on the downstream polarimeter measurement itself have been estimated roughly by taking into account the finite laser spot size and the crossing angle. The variations found are again at a level relevant for permille-level precision. Thus, it will be crucial to monitor the collision parameters in real-time at least to the $10 \%$-level by the forward calorimeters of the main detectors and additional diagnostics like the GamCal and the extraction-line energy spectrometer. With this knowledge, the luminosity-weighted average polarisation at the IP can be extracted from both the upstream and the downstream polarimeter measurements with complementary systematic uncertainties.

In the future, the influence of the spent beam properties on the downstream polarimeter measurement as well as the possibilities to realise the long-range orbit alignment to the tens of micrometer level should be investigated in more detail. Eventually, random misalignments of the beamline elements should be replaced by a proper ground motion model for the actual ILC site in the north of Japan. Many studies performed here depend on the beam energy. In general it is expected that maintaining a permille-level polarisation measurement becomes more difficult with increasing beam energy, while at lower energies, e.g. at the Higgs or $t \bar{t}$ threshold, the impact of the collision effects is reduced. This, however, needs to be quantified. Finally, in order to eliminate the uncertainty from assuming a certain spin configuration at the starting point of the simulation, a full start-to-end spin simulation of the ILC would be highly desirable. 


\section{Acknowledgement}

We thankfully acknowledge the support by the BMBF Verbundforschung "Spin Optimierung" and by the DFG via the Emmy-Noether-Grant Li/1560-1. We thank Karsten Büßer, Mathias Vogt, Peter Schüler, Anthony Hartin, Desmond Barber, Ken Moffeit, Mike Woods and Yuri Nosochkov for many helpful discussions over the last years.

\section{A Appendix}

All results presented in this paper are based on [24]. Some simulations have been rerun with the following modifications:

- The initial spin configurations are slightly different (see section 7.1.2 in [24] and section 5 in this paper). The discrepancy is however much smaller than the difference between the samples TDR and TDR* in section 6, since the angular divergence at the beginning of the lattice is only $1 \mathrm{mrad}$.

- While the collisions are simulated with the TDR beam parameters in this paper (samples TDR and TDR*), the corresponding simulations in [24] are performed with RDR beam parameters and an increased bunch charge, such that the amount of beamstrahlung produced in the collisions is approximately the same as for collisions with TDR beam parameters (sample $\Upsilon_{T D R}$ ).

- For the collision effects, some data samples have been simulated without beamstrahlung and synchrotron radiation. In [24], the initial beam energy spreads are still present (sample no BS,SR), while in this paper also the initial beam energy spreads are set to zero (samples $\mathrm{RDR}_{0}, \mathrm{TDR}_{0}$ and $\mathrm{TDR}_{0}^{*}$ ).

- Misalignments: In contrast to this paper, the corresponding simulations in [24] include bunch rotation at the IP (crab cavities) and energy losses due to synchrotron radiation.

- Measurable Polarisation: In [24], the crossing angle of the laser beam has not been taken into account. Therefore, it is defined by a cut on the radius around the bunch center rather than by a cut on the horizontal distance.

\section{References}

[1] ILC Technical Design Report, 2013, http://www.linearcollider.org/ILC/Publications/ Technical-Design-Report

Volume 1: "Executive Summary," arXiv:1306.6327v1 [physics.ins-det],

Volume 2: "Physics," arXiv:1306.6352v1 [physics.ins-det], 
Volume 3.I: "Accelerator R\&D," arXiv:1306.6353v1 [physics.acc-ph],

Volume 3.II: "Accelerator Baseline Design,"

arXiv:1306.6328v1 [physics.acc-ph],

Volume 4: "Detectors," arXiv:1306.6329v1 [physics.ins-det].

[2] M. S. Amjad et al., "A precise determination of top quark electro-weak couplings at the ILC operating at $\sqrt{s}=500 \mathrm{GeV}, "$ arXiv:1307.8102.

[3] G. Moortgat-Pick et al., "The role of polarized positrons and electrons in revealing fundamental interactions at the Linear Collider," 2005, doi:10.1016/j.physrep.2007.12.003.

[4] C. Bartels, M. Berggren and J. List, "Characterising WIMPs at a future $e^{+} e^{-}$Linear Collider," Eur. Phys. J. C 72 (2012) 2213 [arXiv:1206.6639 [hep-ex]].

[5] I. Bozovic Jelisavcic, S. Lukic, G. Milutinovic Dumbelovi ${ }_{3}$ M. Pandurovic and I. Smiljanic, "Luminosity measurement at ILC," JINST 8 (2013) P08012 [arXiv:1304.4082 [physics.acc-ph]].

[6] K. Mönig, "The use of Positron Polarization for precision Measurements," LC-PHSM2000-059.

[7] I. Marchesini, "Triple Gauge Couplings and Polarization at the ILC and Leakage in a Highly Granular Calorimeter," Ph. D. Thesis, Hamburg University, 2011, DESYTHESIS-11-044.

[8] J. List, "Beam Polarisation and Triple Gauge Couplings in $e^{+} e^{-} \rightarrow W^{+} W^{-}$at the ILC," PoS (EPS-HEP 2013) 233, http://pos.sissa.it/archive/conferences/180/233/EPS-HEP\%202013_233.pdf.

[9] S. Riemann, A. Schalicke and A. Ushakov, "Frequency of Positron Helicity Reversal," arXiv:0903.2366 [physics.ins-det].

[10] S. Riemann, F. Staufenbiel, B. List, N. J. Walker, A. F. Hartin, O. S. Adeyemi, V. S. Kovalenko and L. I. Malysheva et al., "The Design of Spin-Rotator with a Possibility of Helicity Switching for Polarized Positron at the ILC," Conf. Proc. C 1205201 (2012) 1813.

[11] Volume 3.II of [1].

[12] D. Schulte, "Study of Electromagnetic and Hadronic Background in the interaction Region of the TESLA Collider," Ph. D. Thesis, Universität Hamburg, 1996, TESLA 1997-08.

[13] S. Boogert et al., "Polarimeters and Energy Spectrometers for the ILC Beam Delivery System," JINST 4, P10015 (2009), doi:10.1088/1748-0221/4/10/P10015.

[14] ILC Reference Design Report, 2007, http://www. linearcollider.org/ILC/Publications/ Reference-Design-Report 
Volume 1: "Executive Summary," arXiv:0712.1950v1 [physics.acc-ph],

Volume 2: "Physics at the ILC," arXiv:0709.1893v1 [hep-ph],

Volume 3: "Accelerator," arXiv:0712.2361v1 [physics.acc-ph],

Volume 4: "Detectors," arXiv:0712.2356v1 [physics.ins-det].

[15] C. Adolphsen et al., "Design of the ILC crab cavity system," SLAC-PUB-12751.

[16] Volume 3 of [14].

[17] A. Seryi, T. Maruyama and B. Parker, "IR Optimization, DID and anti-DID," SLACPUB-11662.

[18] J. Resta-Lopez, P. N. Burrows, A. F. Hartin, A. Latina and D. Schulte, "Placet Based Start-to-end Simulations of the ILC with Intra-train Fast Feedback System," Conf. Proc. C 0806233 (2008) MOPP027.

[19] I. Bailey, C. Bartels, M. Beckmann, A. Hartin, C. Helebrant, D. Kaefer, J. List and G. Moortgat-Pick, "Time evolution of ground motion-dependent depolarisation at linear colliders," arXiv:1108.6275 [physics.acc-ph].

[20] L. H. Thomas, "The motion of a spinning electron," Nature 117 (1926) 514.

[21] V. Bargmann, L. Michel and V. L. Telegdi, "Precession of the polarization of particles moving in a homogeneous electromagnetic field," Phys. Rev. Lett. 2 (1959) 435.

[22] G. H. Hoffstätter, "High-Energy Polarized Proton Beams - A Modern View," Springer Science+Business Media, LLC, New York, 2006, Springer Tracts in Modern Physics, Vol. 218.

[23] J. C. Smith, "The Preservation of Emittance and Polarization in the International Linear Collider," Ph. D. Thesis, Cornell University, 2007.

[24] M. Beckmann, "Spin Transport at the International Linear Collider and its Impact on the Measurement of Polarization," Ph. D. Thesis, Hamburg University, 2013, DESY-THESIS-13-053.

[25] K. Yokoya, P. Chen, "Depolarization due to Beam-Beam-Interaction in ElectronPositron Linear Colliders," AIP Conf. Proc. 187, 938 (1989), SLAC-PUB-4692.

[26] M. Woods, "Polarimetry at a Future Linear Collider - How precise?," Int. J. Mod. Phys. A15, 2529 (2000), SLAC-PUB-8397, doi:10.1142/S0217751X00002603.

[27] M. Woods, "Machine-Detector Interface Issues for the ILC Polarimeters," SLACPUB-13259, June 2008, doi:10.1142/S0217751X00002603, Proceedings of the workshop "Workshop on Polarisation and Energy Measurements at the ILC" (Zeuthen, Germany, April 2008), https://indico.desy.de/conferenceTimeTable.py? conf Id=585\#all. detailed. 
[28] A. A. Sokolov and I. M. Ternov, "On Polarization and spin effects in the theory of synchrotron radiation," Sov. Phys. Dokl. 8 (1964) 1203 [Dokl. Akad. Nauk Ser. Fiz. 153 (1964) 1052] [Phys. Dokl. 8 (1964) 1203].

[29] K. Yokoya, "User's Manual of CAIN," Version 2.35, April 2003, http://lcdev.kek. jp/ yokoya/CAIN/cain235/CainMan235.pdf.

[30] D. Sagan et al., Bmad, www.lepp.cornell.edu/ dcs/bmad.

[31] D. Sagan, "The Bmad Reference Manual," Revision 16.7 (March 8, 2012), www . lepp. cornell.edu/ dcs/bmad/manual.html.

[32] P. Bambade et al., "Results of the EUROTeV Beam-Beam Simulation (BBSIM) Task," EUROTeV 2008-065.

C. Rimbault et al., "Implementation of depolarization due to beam-beam effects in the beam-beam interaction simulation tool GUINEA-PIG++," EUROTeV 2008-066. G. Le Meur et al., "Description of guineapig++, the $\mathrm{C}++$ upgraded version of the GUINEA-PIG beam-beam simulation program," EUROTeV 2008-067.

Former project website: https://trac.lal.in2p3.fr/GuineaPig.

Current project website:https://savannah.cern.ch/projects/guinea-pig.

[33] D. Schulte, "Beam-beam simulations with GUINEA-PIG," CERN-CLIC-NOTE-387 (1999).

[34] ROOT, versions 5.28 and 5.30, http://root.cern.ch.

[35] U. Fano, J. Op. Soc. Am. 39 (1949) 859.

[36] K. Moffeit, "Downstream Extraction Line Polarimeter," Proceedings of the Workshop on Polarization and Energy Measurements at the ILC, Zeuthen, April 2008, https://indico.desy.de/getFile.py/access?contribId=11\&sessionId= $1 \&$ res $I d=0 \& m a t e r i a l I d=$ paper\&conf $I d=585$.

[37] V. Gharibyan et al., "The TESLA Compton Polarimeter," LC-DET-2001-047, 2001, http://www-flc.desy.de/lcnotes/.

[38] K. Abe et al. [SLD Collaboration], "A High precision measurement of the left-right Z boson cross-section asymmetry," Phys. Rev. Lett. 84 (2000) 5945 [hep-ex/0004026].

[39] C. Bartels, J. Ebert, A. Hartin, C. Helebrant, D. Kafer and J. List, "Design and Construction of a Cherenkov Detector for Compton Polarimetry at the ILC," JINST 7 (2012) P01019 [arXiv:1011.6314 [physics.ins-det]].

[40] B. Vormwald, "From Neutrino Physics to Beam Polarisation - a High Precision Story at the ILC," PhD Thesis, University of Hamburg, 2014, DESY-THESIS-14-006.

[41] D. Angal-Kalinin et al., ILC SB2009_Nov10 lattice, http://projects.astec.ac. uk/ilcdecks. 
[42] B. Aurand, I. Bailey, C. Bartels, G. Blair, A. Brachmann, J. Clarke, L. Deacon and V. Duginov et al., "Executive Summary of the Workshop on Polarization and Beam Energy Measurements at the ILC," arXiv:0808.1638 [physics.acc-ph].

[43] M. Woods et al., "Luminosity, Energy and Polarization Studies for the Linear Collider: Comparing $e^{+} e^{-}$and $e^{-} e^{-}$for NLC and TESLA," 2004, SLAC-PUB-10353.

[44] M. Woods and K. C. Moffeit, "Studies for a downstream Compton polarimeter at the ILC," SLAC-PUB-10669.

[45] S. T. Boogert, F. Cullilnan, A. Lyapin, J. Snuverink, Y. I. Kim, A. Aryshev, Y. Honda and T. Tauchi et al., "ATF2 and LC Cavity Beam Position Monitor Systems," ICFA Beam Dyn. Newslett. 61 (2013) 89.

[46] A. Seryi, T. Maruyama, B. Parker, "IR Optimization, DID and anti-DID," 2006, SLAC-PUB-11662.

[47] H. Abramowicz et al., "Forward Instrumentation for ILC Detectors," JINST 5 (2010) P12002 [arXiv:1009.2433 [physics.ins-det]].

[48] C. Grah and A. Sapronov, "Beam parameter determination using beamstrahlung photons and incoherent pairs," JINST 3 (2008) P10004. 